\title{
Constructing equivariant maps for representations
}

\author{
Stefano Francaviglia
}

February 8, 2020

\begin{abstract}
We show that if $\Gamma$ is a discrete subgroup of the group of the isometries of $\mathbb{H}^{k}$, and if $\rho$ is a representation of $\Gamma$ into the group of the isometries of $\mathbb{H}^{n}$, then any $\rho$-equivariant map $F: \mathbb{H}^{k} \rightarrow \mathbb{H}^{n}$ extends to the boundary in a weak sense in the setting of Borel measures. As a consequence of this fact, we obtain an extension of a result of Besson, Courtois and Gallot about the existence of volume non-increasing, equivariant maps. Moreover, under an additional hypothesis, we show that the weak extension we obtain is actually a measurable $\rho$-equivariant map $\partial \mathbb{H}^{k} \rightarrow \overline{\mathbb{H}}^{n}$. We use this fact to obtain measurable versions of Cannon-Thurston-type results for equivariant Peano curves.
\end{abstract}

\section{Introduction}

Let $\Gamma$ be a discrete subgroup of $\operatorname{Isom}\left(\mathbb{H}^{\mathrm{k}}\right)$ and let $\rho: \Gamma \rightarrow \operatorname{Isom}\left(\mathbb{H}^{\mathrm{n}}\right)$ be a representation. Then, it is easy to construct a piecewise smooth map $D: \mathbb{H}^{k} \rightarrow \mathbb{H}^{n}$ which is $\rho$-equivariant, that is $D(\gamma x)=\rho(\gamma) D(x)$ for all $\gamma \in \Gamma$ and $x \in \mathbb{H}^{k}$, and the problem arises of whether such a map continuously extends to the boundaries of the hyperbolic spaces (this is a key step in the proofs of rigidity results for hyperbolic manifolds, see for example [Thu79, BP92, Dun99, Fra04, Kla ). In some cases, for example if the representation is not discrete, such an extension is not possible. Moreover, in general it is hard even to construct a $\rho$-equivariant map between the boundaries with some regularity properties like continuity and measurability. If $\Gamma<\operatorname{Isom}\left(\mathbb{H}^{2}\right)$ is a surface group and if $\rho: \Gamma \rightarrow \operatorname{Isom}\left(\mathbb{H}^{3}\right)$ is an isomorphism such that $\mathbb{H}^{3} / \rho(\Gamma)$ is an hyperbolic 3-manifold, under certain assumptions, Cannon and Thurston [CT89] and Minsky Min94, proved the existence of a continuous, 
$\rho$-equivariant, surjective map $\partial \mathbb{H}^{2} \rightarrow \partial \mathbb{H}^{3}$, and Soma Som95] proved that, outside zero-measure sets, such a map is a homeomorphism.

In this paper we show that any $\rho$-equivariant map $\mathbb{H}^{k} \rightarrow \mathbb{H}^{n}$ weakly extends to the boundaries in the setting of Borel measures. Namely, the extension of a map from $\mathbb{H}^{k}$ to $\mathbb{H}^{n}$ will be a family $\left\{\lambda_{z}\right\}_{z \in \partial \mathbb{H}^{k}}$ of Borel measures on $\overline{\mathbb{H}}^{n}$.

We state now the main consequences of this fact, referring to Section 2 for precise definitions.

After having introduced the notion of family of developing measures, which generalizes the one of measurable $\rho$-equivariant map, we prove the following existence result.

Theorem 1.1 (Existence of developing measures) Let $\Gamma<\operatorname{Isom}\left(\mathbb{H}^{\mathrm{k}}\right)$ be an infinite, non-elementary discrete group. Let $\rho: \Gamma \rightarrow \operatorname{Isom}\left(\mathbb{H}^{\mathrm{n}}\right)$ be a representation. Then, a family of developing measures for $\rho$ exists.

Then, modifying the construction of Besson Courtois and Gallot (see BCG95, BCG96, BCG99]), we get the following Theorem.

Theorem 1.2 (B-C-G-natural maps) Let $\Gamma<\operatorname{Isom}\left(\mathbb{H}^{\mathrm{k}}\right)$ be an infinite discrete group. Let $\rho: \Gamma \rightarrow \operatorname{Isom}\left(\mathbb{H}^{\mathrm{n}}\right)$ be a representation whose image is non-elementary. Then, there exists a map $F: \mathbb{H}^{k} \rightarrow \mathbb{H}^{n}$, called natural map, such that:

1. F is smooth.

2. $F$ is $\rho$-equivariant, i.e. $F(\gamma x)=\rho(\gamma) F(x)$ for all $x \in \mathbb{H}^{k}$ and $\gamma \in \Gamma$.

3. For all $p \geq 3, \operatorname{Jac}_{p} F(x) \leq\left(\frac{\delta(\Gamma)}{p-1}\right)^{p}$.

4. If $\left\|d_{x} F\left(u_{1}\right) \wedge \cdots \wedge d_{x} F\left(u_{p}\right)\right\|=\left(\frac{\delta(\Gamma)}{p-1}\right)^{p}$ for an orthonormal $p$-frame $u_{1}, \ldots, u_{p}$ at $x \in \mathbb{H}^{k}$, then the restriction of $d_{x} F$ to the subspace generated by $u_{1}, \ldots, u_{p}$ is a homothety.

An immediate corollary of Theorem 1.2 is the following fact.

Corollary 1.3 Let $X, Y$ be two compact hyperbolic manifold of (possibly different) dimension at least tree. Then in each homotopy class of maps $f: Y \rightarrow X$ there exists a smooth map $F: Y \rightarrow X$ such that $|\operatorname{Jac}(F)| \leq 1$. Moreover, if $|\operatorname{JacF}(y)|=1$, then $d_{y} F$ is an isometry. 
Corollary 1.3 follows from Theorem 1.2 because any $f: Y \rightarrow X$ is homotopic to the natural map corresponding to the representation $f_{*}$. Such a map has the requested properties because, as we will see in Lemma 2.5. we have $\delta\left(\pi_{1}(Y)\right) \leq \operatorname{dim}(Y)$.

Theorem 1.2 and Corollary 1.3 were proved by Besson Courtois and Gallot in the special case in which $\rho$ is discrete and faithful and both $\Gamma$ and $\rho(\Gamma)$ are convex co-compact ([BCG99, Théorème1.10 and Théorème 1.2]).

In our setting, Theorem 1.2 will follow directly from Theorem 1.1 and the following

Theorem 1.4 Let $\Gamma<\operatorname{Isom}\left(\mathbb{H}^{\mathrm{k}}\right)$ be an infinite discrete group. Let $\rho: \Gamma \rightarrow$ Isom $\left(\mathbb{H}^{\mathrm{n}}\right)$ be a representation whose image is non-elementary. If there exists a family $\left\{\lambda_{z}\right\}$ of developing measures for $\rho$, then a natural map exists.

In [BCG99], for $\varepsilon>0$ the authors construct a smooth $\rho$-equivariant map $F_{\varepsilon}: \mathbb{H}^{k} \rightarrow \mathbb{H}^{n}$ such that for all $p \geq 3, \operatorname{Jac}_{p} F_{\varepsilon}(x) \leq\left(\frac{\delta(\Gamma)(1+\varepsilon)}{p-1}\right)^{p}$. We call such maps $\varepsilon$-natural maps. We will see that the natural map we construct is actually the limit of a sequence of such $\varepsilon$-natural maps.

Proposition 1.5 In the hypotheses of Theorem 1.2, there exists a family $\left\{F_{\varepsilon}\right\}$ of $\varepsilon$-natural maps (constructed as in [BCG99]) and a sequence $\varepsilon_{i} \rightarrow 0$ such that $F_{\varepsilon_{i}}$ converges to the natural map $F$.

The proofs of Theorem 1.1 and Proposition 1.5 both start with a $\rho$ equivariant map $D: \mathbb{H}^{k} \rightarrow \mathbb{H}^{n}$. While the natural map $F$ does not depend on $D$, the maps $F_{\varepsilon}$ 's can be constructed in such a way to keep memory of $D$. More precisely, the construction of the $\varepsilon$-natural maps depends on the choice of a probability measure on a fundamental domain for the action of $\Gamma$, and they depends on the restriction of $D$ to the support of such measure. This is useful to study non-compact manifolds. For example, suppose that $f: M \rightarrow N$ is a proper map between complete non-compact hyperbolic manifolds. If $D$ is the lift of $f$ to the universal covers, then the natural maps $F_{\varepsilon}$ descend to maps $f_{\varepsilon}: M \rightarrow N$, and one can show that, if one used the volume-measure to construct such maps, then they are proper (see [Sou00]).

Results such as Theorem 1.2 and Proposition 1.5 will be used in [FK04 to prove rigidity results for Fuchsian representations. More precisely, in FK04] the authors will show

Theorem 1.6 (Rigidity of representations) Let $M$ be a complete hyperbolic $k$-manifold of finite volume. Let $\rho: \pi_{1}(M) \rightarrow \operatorname{Isom}\left(\mathbb{H}^{\mathrm{n}}\right)$ be a representation. Then $\operatorname{vol}(\rho) \leq \operatorname{vol}(M)$ and equality holds if and only if $\rho$ is a discrete 
and faithful representation into the group of isometries of a $k$-dimensional hyperbolic subspace of $\mathbb{H}^{n}$.

Then, we investigate the extension of the natural maps to the boundaries. Theorem 1.1 is a kind of weak extension result. Under some additional hypotheses, we show a result of strong, measurable extension (the measures we consider on $\partial \mathbb{H}^{k}$ are the Patterson-Sullivan measures, see Section 2).

Theorem 1.7 (Measurable extension) Let $\Gamma<\operatorname{Isom}\left(\mathbb{H}^{\mathrm{k}}\right)$ be a discrete group and let $\rho: \Gamma \rightarrow \operatorname{Isom}\left(\mathbb{H}^{\mathrm{n}}\right)$ be a representation whose image is nonelementary. Suppose that there exists a family $\left\{\lambda_{z}\right\}_{z \in \partial \mathbb{H}^{k}}$ of developing measures for $\rho$ such that for almost all $z$, the measure $\lambda_{z}$ is not the sum of two Dirac deltas with equal weights. Then, the natural map $F$ constructed using $\left\{\lambda_{z}\right\}$ extends to the conical limit set. More precisely, there exists a measurable, $\rho$-equivariant map $\bar{F}: \overline{\mathbb{H}}^{k} \rightarrow \overline{\mathbb{H}}^{n}$ which agrees with $F$ on $\mathbb{H}^{k}$ and such that, for almost all $\omega$ in the conical limit set, if $\xi \in \mathbb{H}^{k}$ and $\left\{\gamma_{n} \xi\right\}$ is a sequence conically converging to $\omega$, then $F\left(\gamma_{n} x\right) \rightarrow \bar{F}(\omega)$ for all $x \in \mathbb{H}^{k}$.

Theorem [1.7 in particular applies to the case of fundamental groups of hyperbolic manifolds. More precisely, we prove

Theorem 1.8 Let $\Gamma<\operatorname{Isom}\left(\mathbb{H}^{\mathrm{k}}\right)$ be a discrete group such that $\delta(\Gamma)=k-1$ and which diverges at $k-1$. Let $\rho: \Gamma \rightarrow \operatorname{Isom}\left(\mathbb{H}^{\mathrm{n}}\right)$ be a representation whose image is not elementary and let $\left\{\lambda_{z}\right\}_{z \in \partial \mathbb{H}^{k}}$ be a family of developing measures for $\rho$. Then, for almost all $z$, the measure $\lambda_{z}$ is not the sum of two Dirac deltas with equal weights.

Such results allow us to establish measurable versions of Cannon-Thurstontype results for equivariant Peano curves. In particular, we get the following results.

Corollary 1.9 (Measurable, equivariant Peano curves) Let $\mathbb{H}^{k} / \Gamma$ be a complete hyperbolic manifold of finite volume and let $\rho: \Gamma \rightarrow \operatorname{Isom}\left(\mathbb{H}^{\mathrm{n}}\right)$ be a representation whose image is not elementary. Then there exists a measurable $\rho$-equivariant map $\bar{F}: \partial \mathbb{H}^{k} \rightarrow \overline{\mathbb{H}}^{n}$ which is the extension of a natural map. Moreover, if $\rho$ is discrete and faithful, then the image of $\bar{F}$ is contained in $\partial \mathbb{H}^{n}$.

Theorem 1.10 (Equivariant inverse of measurable Peano curves) Let $\Gamma<\operatorname{Isom}\left(\mathbb{H}^{\mathrm{k}}\right)$ and $\Gamma^{\prime}<\operatorname{Isom}\left(\mathbb{H}^{\mathrm{n}}\right)$ be non-elementary discrete groups, with $\delta(\Gamma)=k-1$ and $\delta\left(\Gamma^{\prime}\right)=n-1$, such that they diverge respectively at $k-1$ and $n-1$. Let $\rho: \Gamma \rightarrow \Gamma^{\prime}$ be an isomorphism. 
Then, there exist measurable maps $\bar{F}: \partial \mathbb{H}^{k} \rightarrow \partial \mathbb{H}^{n}$ and $\bar{G}: \partial \mathbb{H}^{n} \rightarrow \partial \mathbb{H}^{k}$ which are respectively $\rho$ and $\rho^{-1}$ equivariant. Moreover, almost everywhere

$$
\bar{F} \circ \bar{G}=\operatorname{Id}_{\mathbb{H}^{k}} \quad \bar{G} \circ \bar{F}=\operatorname{Id}_{\mathbb{H}^{n}} .
$$

Corollary 1.11 ([CT89, Min94, Som95]) Let $M$ be a compact hyperbolic 3-manifold fibered over the circle with fiber a surface $F$. Let $\pi_{1}(F) \simeq \Gamma<$ $\operatorname{Isom}\left(\mathbb{H}^{2}\right)$ and let $\rho: \Gamma \rightarrow \operatorname{Isom}\left(\mathbb{H}^{3}\right)$ be the representation induced by the inclusion $\pi_{1}(F) \hookrightarrow \pi_{1}(M)$. Then, there exist measurable maps $\bar{F}: \partial \mathbb{H}^{2} \rightarrow$ $\partial \mathbb{H}^{3}$ and $\bar{G}: \partial \mathbb{H}^{3} \rightarrow \partial \mathbb{H}^{2}$ which are respectively $\rho$ and $\rho^{-1}$ equivariant. Moreover, almost everywhere

$$
\bar{F} \circ \bar{G}=\operatorname{Id}_{\mathbb{H}^{2}} \quad \bar{G} \circ \bar{F}=\operatorname{Id}_{\mathbb{H}^{3}} .
$$

The paper is structured as follows.

In Section 2 we give definitions and we prove some preliminary facts. We recall the definition of Patterson-Sullivan measures and of barycentre of a measure. We introduce here the notion of family of developing measures, that generalises the one of $\rho$-equivariant map.

In Section [3, we prove Theorem 1.4 We do it extending the B-C-Gtechnique for constructing natural maps by replacing the role of equivariant maps with a family of developing measures.

In Sections 4, we give a proof of Theorem 1.1, showing that any equivariant map weakly extend to the boundary.

In Section 5, we show how to construct sequences of measures approximating Patterson-Sullivan measures. We will use such sequences to prove Proposition 1.5.

In Section 66, we prove Theorem 1.7. The techniques we use come from classical harmonic analysis. Namely, the proof of Theorem [1.7] goes as the proof of the existence of harmonic extensions of measurable maps.

In Section 7 we prove a kind of regularity theorem, showing that, under some additional hypotheses, the weak extension obtained in Section 4 actually is a measurable map. We prove here Theorems 1.8 and 1.10 and Corollaries 1.9 and 1.11 .

Acknowledgements. I would like to thank Benjamin Klaff and Juan Souto for the stimulating and interesting conversations this work started with. I also thank Carlo Mantegazza and Joan Porti for their fundamental help. This work was partially supported by an INdAM fellowship. 


\section{Definitions, notation and preliminary facts}

First of all, we recall some definitions.

Definition 2.1 A subgroup $\Gamma<\operatorname{Isom}\left(\mathbb{H}^{\mathrm{k}}\right)$ is said non-elementary if any $\Gamma$ invariant, non-empty set $A \subset \partial \mathbb{H}^{k}$ contains at least three points. Otherwise, $\Gamma$ is said elementary.

Definition 2.2 Let $F: \mathbb{H}^{k} \rightarrow \mathbb{H}^{n}$ be a smooth map. The p-Jacobian $\mathrm{Jac}_{p} F$ of $F$ is defined by

$$
\mathrm{Jac}_{p} F(x)=\sup \left\|d_{x} F\left(u_{1}\right) \wedge, \ldots, \wedge d_{x} F\left(u_{p}\right)\right\|_{\mathbb{H}^{n}},
$$

where $\left\{u_{i}\right\}_{i=1}^{p}$ varies on the set of orthonormal $p$-frames at $x \in \mathbb{H}^{k}$.

Definition 2.3 Let $(X, g)$ be a complete Riemannian manifold and let $\Gamma$ be a group of isometries of $X$. We denote by $\delta(\Gamma)$ the critical exponent of the Poincaré series of $\Gamma$, that is:

$$
\delta(\Gamma)=\inf \left\{s>0: \sum_{\gamma \in \Gamma} e^{-s d(x, \gamma x)}<+\infty\right\}
$$

where $d(\cdot, \cdot)$ denotes the distance induced by $g$ on $X$ and $x$ is a point of $X$.

It is readily checked that $\delta(\Gamma)$ does not depend on $x$. We notice that, when $X=\mathbb{H}^{k}$, the critical exponent is the Hausdorff dimension of the limit set of $\Gamma$. Moreover, $\delta(\Gamma)$ can be computed by

$$
\delta(\Gamma)=\lim _{R \rightarrow \infty} \frac{1}{R} \log (\#\{\gamma \in \Gamma: d(\gamma O, O)<R\}) .
$$

We refer to [Nic89, Sul84, Yue96a, for details.

Definition 2.4 Let $(X, g)$ be a complete Riemannian manifold and let $\Gamma$ be a group of isometries of $X$. We say that $\Gamma$ diverges at $\delta(\Gamma)$ if

$$
\lim _{s \rightarrow \delta(\Gamma)^{+}} \sum_{\gamma \in \Gamma} e^{-s d(x, \gamma x)}=+\infty .
$$

The following lemma is an immediate consequence of [Nic89, Theorem 1.6.1], Nic89, Theorem 1.6.3] and [Nic89, Corollary 3.4.5].

Lemma 2.5 Let $\Gamma$ be a infinite, non-elementary discrete subgroup of $\operatorname{Isom}\left(\mathbb{H}^{\mathrm{k}}\right)$, then

$$
0<\delta(\Gamma) \leq k-1
$$

Moreover, if $\mathbb{H}^{k} / \Gamma$ has finite volume, then $\delta(\Gamma)=k-1$ and $\Gamma$ diverges at $\delta(\Gamma)$. 
Notation. For the rest of the paper we fix the following notation. $\Gamma$ will be an infinite discrete subgroup of $\operatorname{Isom}\left(\mathbb{H}^{\mathrm{k}}\right)$, and

$$
\rho: \Gamma \rightarrow \operatorname{Isom}\left(\mathbb{H}^{\mathrm{n}}\right)
$$

will be a representation. We fix base-points in $\mathbb{H}^{k}$ and $\mathbb{H}^{n}$, both denoted by $O$. We denote by $B_{K}$ and $B_{N}$ the Busemann functions, respectively of $\mathbb{H}^{k}$ and $\mathbb{H}^{n}$, normalised at $O$. Namely, for $x \in \mathbb{H}^{k}$ and $\theta \in \partial \mathbb{H}^{k}$ (resp. $\mathbb{H}^{n}$ and $\left.\partial \mathbb{H}^{n}\right)$ we set

$$
B_{K}(x, \theta)=\lim _{t \rightarrow \infty}\left(d_{\mathbb{H}^{k}}\left(x, \gamma_{\theta}(t)\right)-t\right),
$$

where $\gamma_{\theta}$ is the geodesic ray from $O$ to $\theta$, parameterised by arc length. We denote by $\pi_{K}$ (respectively $\pi_{N}$ ) the projection of $\overline{\mathbb{H}}^{k} \times \overline{\mathbb{H}}^{n}$ to $\overline{\mathbb{H}}^{k}$ (resp. $\overline{\mathbb{H}}^{n}$ ):

$$
\pi_{K}: \overline{\mathbb{H}}^{k} \times \overline{\mathbb{H}}^{n} \rightarrow \overline{\mathbb{H}}^{k}
$$

Finally, we fix a piecewise smooth $\rho$-equivariant map

$$
D: \mathbb{H}^{k} \rightarrow \mathbb{H}^{n} .
$$

We notice that such a map can be easily constructed by triangulating a fundamental domain for $\Gamma$ and then arguing by induction on the $i$-skeleta.

Patterson-Sullivan measures. A fundamental tool for our purpose is the family of Patterson-Sullivan measures. We recall the main results we need, which we summarise in Theorem 2.6, referring to BCG99, Nic89, Chap. 3, 4] and Yue96a, Yue96b for proofs and details.

Theorem 2.6 Let $\Gamma$ be an infinite, non-elementary discrete subgroup of Isom $\left(\mathbb{H}^{\mathrm{k}}\right)$ with critical exponent $\delta(\Gamma)$. For all $x \in \mathbb{H}^{k}$ there exists a positive Borel measure $\mu_{x}$ of finite, non-zero mass such that, for all $x, y \in \mathbb{H}^{k}$ and $\gamma \in \Gamma$ :

1. The measures $\mu_{x}$ and $\mu_{y}$ are in the same density class of measures and are concentrated on $\partial \mathbb{H}^{k}$.

2. The measure $\mu_{x}$ satisfies

$$
d \mu_{x}(\theta)=e^{-\delta(\Gamma) B_{K}(x, \theta)} d \mu_{O}(\theta)
$$

where $\theta \in \partial \mathbb{H}^{k}$.

3. The measures $\mu_{x}$ are $\Gamma$-equivariant, that is

$$
\mu_{\gamma x}=\gamma_{*} \mu_{x}
$$


Proof. We only sketch the proof, recalling the construction of the PattersonSullivan measures because we will explicitly use it in the following.

For all $s>\delta(\Gamma)$ let

$$
c(s)=\sum_{\gamma \in \Gamma} e^{-s d(O, \gamma O)}
$$

where $d(\cdot, \cdot)$ denotes the hyperbolic distance of $\mathbb{H}^{k}$. For simplicity, here we stick to the case that $\Gamma$ diverges at $\delta(\Gamma)$ (this happens for example if $\Gamma$ is geometrically finite, see [Nic89, p. 87]). For any $x \in \mathbb{H}^{k}$ and $s>\delta(\Gamma)$, define

$$
\mu_{x}^{s}=\frac{1}{c(s)} \sum_{\gamma \in \Gamma} e^{-s d(x, \gamma O)} \delta_{\gamma O}
$$

where $\delta_{\gamma O}$ is the Dirac measure concentrated on $\gamma O$. For $s>\delta(\Gamma), \mu_{x}^{s}$ is a well-defined positive Borel measure on $\mathbb{H}^{k} \subset \overline{\mathbb{H}}^{k}$. It can be shown that for $s \rightarrow \delta(\Gamma)^{+}$, the measures $\mu_{x}^{s}$ weakly converge to a positive Borel measure $\mu_{x}$ on $\overline{\mathbb{H}}^{k}$. More precisely, for all $x \in \mathbb{H}^{k}$ and $\varphi \in C\left(\overline{\mathbb{H}}^{k}\right)$

$$
\int_{\overline{\mathbb{H}}^{k}} \varphi d \mu_{x}^{s} \rightarrow \int_{\overline{\mathbb{H}}^{k}} \varphi d \mu_{x}
$$

Moreover, the fact that $\lim _{s \rightarrow \delta(\Gamma)^{+}} c(s)=+\infty$ easily implies that $\mu_{x}$ is concentrated on the boundary (in fact $\mu_{x}$ is concentrated on the limit set of $\Gamma$ ).

BARYCENTRE OF A MEASURE. We recall now the definition and the main properties of the barycentre of a measure. We refer the reader to [DE86] and [BCG95] for complete proofs and details.

Let $\beta$ be a positive Borel measure on $\partial \mathbb{H}^{n}$ of finite mass. Define the function $\mathcal{B}_{\beta}: \mathbb{H}^{n} \rightarrow \mathbb{R}$ by

$$
\mathcal{B}_{\beta}: y \mapsto \int_{\partial \mathbb{H}^{n}} B_{N}(y, \theta) d \beta(\theta)
$$

Since we are working in the hyperbolic space, the Busemann functions are convex. Thus, its $\beta$-average $\mathcal{B}_{\beta}$ is strictly convex, provided that $\beta$ is not the sum of two deltas. Moreover, one can show that $\mathcal{B}_{\beta}(y) \rightarrow \infty$ as $y$ approaches $\partial \mathbb{H}^{n}$. It follows that $\mathcal{B}$ has a unique minimum in $\mathbb{H}^{n}$.

Definition 2.7 For any positive Borel measure $\beta$ on $\partial \mathbb{H}^{n}$ of finite mass which is not concentrated on two points, we define the barycentre $\operatorname{bar}(\beta)$ of $\beta$ as the unique minimum point of the function $y \mapsto \int_{\partial \mathbb{H}^{n}} B_{N}(y, \theta) d \beta(\theta)$. 
We refer to [DE86], [BCG95] and [BCG96] for a proof of the following lemma.

Lemma 2.8 The barycentre of a measure $\beta$ satisfies the following properties:

1. The barycentre is characterised by the equation

$$
\int_{\partial \mathbb{H}^{n}} d B_{N(\operatorname{bar}(\beta), \theta)}(\cdot) d \beta(\theta)=0 .
$$

2. The barycentre is $\operatorname{Isom}\left(\mathbb{H}^{\mathrm{n}}\right)$-equivariant, that is, for any $g \in \operatorname{Isom}\left(\mathbb{H}^{\mathrm{n}}\right)$

$$
\operatorname{bar}\left(g_{*} \beta\right)=g(\operatorname{bar}(\beta)) \text {. }
$$

3. The barycentre is continuous respect the weak convergence of measures. That is, if $\beta_{i} \rightarrow \beta$, then $\operatorname{bar}\left(\beta_{i}\right) \rightarrow \operatorname{bar}(\beta)$.

The first property follows from the definition after differentiating the function $\mathcal{B}_{\beta}$. The equivariance follows from the properties of the Busemann functions. The continuity can be easily proved using that, if $\beta_{i} \rightarrow \beta$, then $\mathcal{B}_{\beta_{i}}$ and $d \mathcal{B}_{\beta_{i}}$ point-wise converge to $\mathcal{B}_{\beta}$ and $d \mathcal{B}_{\beta}$ respectively.

Remark 2.9 If $\beta=a \delta_{\theta_{1}}+b \delta_{\theta_{2}}$, with $0<a<b$, then it can be checked that the minimum of $\mathcal{B}_{\beta}$ is the point $\theta_{2} \in \partial \mathbb{H}^{n}$. Thus, one can define the barycentre of a measure $\beta$ whenever $\beta$ is not the sum of two deltas with the same weights. Note that, since the barycentre of a measure concentrated on two points belongs to $\partial \mathbb{H}^{n}$, equation 1 of Lemma 2.8 makes no sense for such measures.

DEVEloping MEASURES. We introduce now the notion of family of developing measures for $\rho$, which extends the one of $\rho$-equivariant map. We recall that $\left\{\mu_{x}\right\}$ is the family of Patterson-Sullivan measures.

Definition 2.10 A family of developing measures for $\rho$ is a set $\left\{\lambda_{z}\right\}_{z \in \partial \mathbb{H}^{k}}$ of positive Borel measures on $\partial \mathbb{H}^{n}$, of finite mass, and such that:

1. The measures $\lambda_{z}$ 's are $\rho$-equivariant, that is, for $\mu_{O}$-almost all $z$ and all $\gamma \in \Gamma$

$$
\lambda_{\gamma z}=\rho(\gamma)_{*} \lambda_{z}
$$


2. For any $\varphi \in C\left(\partial \mathbb{H}^{n}\right)$, the function

$$
z \mapsto \int_{\partial \mathbb{H}^{n}} \varphi(\theta) d \lambda_{z}(\theta)
$$

is $\mu_{O}$-integrable (whence, by points 1] and [2 of Theorem [2.6, it is $\mu_{x^{-}}$ integrable for all $x$ ).

3. The function $z \mapsto\left\|\lambda_{z}\right\|$ belongs to $L^{\infty}\left(\partial \mathbb{H}^{k}, \mu_{O}\right)$.

4. For $\mu_{O}$-almost $z \in \partial \mathbb{H}^{k},\left\|\lambda_{z}\right\|>0$.

As an example, consider a $\mu_{O}$-measurable $\rho$-equivariant map $\bar{D}: \partial \mathbb{H}^{k} \rightarrow$ $\partial \mathbb{H}^{n}$. Then the family $\left\{\lambda_{z}=\delta_{\bar{D}(z)}\right\}$, where $\delta_{\bar{D}(z)}$ is the Dirac measure, is a family of developing measures for $\rho$. In this sense the notion of developing measures extends the one of equivariant map.

Convolutions of measures. Let $X, Y$ be topological spaces and let $\mu$ be a Borel measure on $X$. Let $\left\{\alpha_{x}\right\}_{x \in X}$ be a family of Borel measures on $Y$ such that for each $\varphi \in C_{0}(Y)$ the function $x \mapsto \int_{Y} \varphi d \alpha_{x}$ is $\mu$-integrable. The convolution $\mu *\left\{\alpha_{x}\right\}$ is the Borel measure on $Y$ defined by

$$
\int_{Y} \varphi(y) d\left(\mu *\left\{\alpha_{x}\right\}\right)=\int_{X}\left(\int_{Y} \varphi(y) d \alpha_{x}(y)\right) d \mu(x)
$$

for any $\varphi \in C_{0}(Y)$. Similarly, we define the product $\mu \times\left\{\alpha_{x}\right\}$ on $X \times Y$ by

$$
\int_{Y} \varphi(x, y) d\left(\mu \times\left\{\alpha_{x}\right\}\right)=\int_{X}\left(\int_{Y} \varphi(x, y) d \alpha_{x}(y)\right) d \mu(x) .
$$

The measure $\mu *\left\{\alpha_{x}\right\}$ is the $\mu$-average of the $\alpha_{x}$ 's. Moreover, if $\pi: X \times Y \rightarrow Y$ is the projection, then $\mu *\left\{\alpha_{x}\right\}=\pi_{*}\left(\mu \times\left\{\alpha_{x}\right\}\right)$.

We say that a sequence of measures $\left\{\mu_{i}\right\}$ weakly converges to $\mu$ if, for any continuous function $f$ with compact support, $\int f d \mu_{i} \rightarrow \int f d \mu$. The proof of following lemmas are left to the reader.

Lemma 2.11 Suppose that $\left\{\mu_{i}\right\}$ is a sequence of measures on $X$, weakly converging to $\mu$. If for each $\varphi \in C_{0}(Y)$ the function $x \mapsto \int_{Y} \varphi d \alpha_{x}$ belongs to $C_{0}(X)$, then the sequence $\mu_{i} *\left\{\alpha_{x}\right\}$ weakly converges to $\mu *\left\{\alpha_{x}\right\}$.

Lemma 2.12 Let $Z$ be a topological space and let $\left\{\nu_{y}\right\}_{y \in Y}$ be a family of Borel measures on $Z$ such that for all $\psi \in C_{0}(z)$ the function $y \mapsto \int_{Z} \psi d \nu_{y}$ is $\alpha_{x}$-integrable for $\mu$-almost all $x$ and $x \mapsto \int_{Y} \int_{Z} \psi d \nu_{y} d \alpha_{x}$ is $\mu$-measurable. Then

$$
\mu *\left\{\alpha_{x} *\left\{\nu_{y}\right\}\right\}=\left(\mu *\left\{\alpha_{x}\right\}\right) *\left\{\nu_{y}\right\}
$$


By Lemma 2.12 we can omit the parentheses and write $\mu *\left\{\alpha_{x}\right\} *\left\{\nu_{y}\right\}$.

Some ReMARKS ON THE HYPOTHESES OF THEOREMS 1.1, 1.2 AND 1.4, First of all, we notice that the hypotheses of such theorems can be relaxed by replacing the spaces $\mathbb{H}^{k}$ and $\mathbb{H}^{n}$ with Riemannian manifolds with suitable bounds on the curvatures. We refer the reader to BCG99] for further details on that direction.

Moreover, even if the hypothesis that the image of $\rho$ is non-elementary is crucially used in Corollary 3.4. it is not strictly necessary. More precisely, suppose that $\rho(\Gamma)$ is elementary. Then there exists a $\rho(\Gamma)$-invariant set $A \subset$ $\partial \mathbb{H}^{n}$ with either one or two points.

In the latter case, there exists a whole geodesic $c$ in $\mathbb{H}^{n}$ which is $\rho(\Gamma)$ invariant and it is easy to see that a natural map whose image is contained in $c$ exists. Therefore, Theorem 1.2 is true in this case.

In the former case, we can suppose that, in the half-space model $\mathbb{H}^{n-1} \times \mathbb{R}^{+}$ of $\mathbb{H}^{n}$, the point $\infty$ is fixed by $\rho(\Gamma)$. Then, one can easily construct a $\rho$ equivariant map $D$ whose image is contained in the horosphere $\{(z, 1): z \in$

$\mathbb{R}^{n-1}$ \}. Thus, if $\operatorname{Jac}_{p} D(x)$ is bounded, then Theorem 1.2 is proved by raising $D$ to a sufficiently high horosphere. It follows that, if $\mathbb{H}^{k} / \Gamma$ is compact, or simply if $\mathbb{H}^{k} / \Gamma$ retracts to a compact set (and this is the case if for example $\Gamma$ is geometrically finite), then Theorem 1.2 is true even if $\rho(\Gamma)$ has a fixed point.

\section{Construction of natural maps}

This section is entirely devoted to prove Theorem 1.4. For this section we keep the notation fixed in Section 2, recalling that the group $\rho(\Gamma)$ is supposed to be non-elementary, and we fix a family

$$
\left\{\lambda_{z}\right\}_{z \in \partial \mathbb{H}^{k}}
$$

of developing measures for $\rho$. The idea of the proof is to use the developing measures to push-forward the Patterson-Sullivan measures $\mu_{x}$ 's to measures $\beta_{x}$ 's on $\partial \mathbb{H}^{n}$, and define the natural map by

$$
x \mapsto \mu_{x} \mapsto \beta_{x} \mapsto \operatorname{bar}\left(\beta_{x}\right) .
$$

Then, the properties of the natural map will follows as in BCG99.

The push-forward the measures $\mu_{x}$ 's is defined as follows. For each $x \in$ $\mathbb{H}^{k}$, define $\beta_{x}$ as the positive Borel measure on $\partial \mathbb{H}^{n}$ given by $\beta_{x}=\mu_{x} *\left\{\lambda_{z}\right\}$. 
Namely, for all $\varphi \in C\left(\partial \mathbb{H}^{n}\right)$

$$
\begin{aligned}
\int_{\partial \mathbb{H}^{n}} \varphi(y) d \beta_{x}(y) & =\int_{\partial \mathbb{H}^{k}}\left(\int_{\partial \mathbb{H}^{n}} \varphi(y) d \lambda_{z}(y)\right) d \mu_{x}(z) \\
& =\int_{\partial \mathbb{H}^{k}}\left(\int_{\partial \mathbb{H}^{n}} \varphi(y) d \lambda_{z}(y)\right) e^{-\delta(\Gamma) B_{K}(x, z)} d \mu_{O}(z) .
\end{aligned}
$$

Note that the measure $\beta_{x}$ is well-defined and has finite mass because of conditions (21) and (3) of Definition 2.10. Moreover, since $\beta_{x}=\pi_{N *}\left(\mu_{x} \times\right.$ $\left\{\lambda_{z}\right\}$ ), if the family $\left\{\lambda_{z}\right\}$ is of the form $\left\{\delta_{\bar{D}(z)}\right\}$ for a $\mu_{O}$-measurable function $\bar{D}: \partial \mathbb{H}^{k} \rightarrow \partial \mathbb{H}^{n}$, then $\beta_{x}=\bar{D}_{*} \mu_{x}$.

Lemma 3.1 The family of measures $\left\{\beta_{x}\right\}_{x \in \mathbb{H}^{k}}$ is $\rho$-equivariant, that, is for all $x \in \mathbb{H}^{k}$ and $\gamma \in \Gamma$

$$
\beta_{\gamma x}=\rho(\gamma)_{*} \beta_{x}
$$

Proof. For any $\varphi \in C\left(\partial \mathbb{H}^{n}\right)$

$$
\begin{aligned}
& \int_{\partial \mathbb{H}^{n}} \varphi(y) d \beta_{\gamma x}(y)=\int_{\partial \mathbb{H}^{k}}\left(\int_{\partial \mathbb{H}^{n}} \varphi(y) d \lambda_{z}(y)\right) d \mu_{\gamma x}(z) \\
= & \int_{\partial \mathbb{H}^{k}}\left(\int_{\partial \mathbb{H}^{n}} \varphi(y) d \lambda_{\gamma z}(y)\right) d \mu_{x}(z) \\
= & \int_{\partial \mathbb{H}^{k}}\left(\int_{\partial \mathbb{H}^{n}} \varphi(\rho(\gamma) y) d \lambda_{z}(y)\right) d \mu_{x}(z)=\int_{\partial \mathbb{H}^{n}} \varphi(y) d\left(\rho(\gamma)_{*} \beta_{x}\right)(y) .
\end{aligned}
$$

Lemma 3.2 For all $x, y \in \mathbb{H}^{k}$, the measures $\beta_{x}$ and $\beta_{y}$ are in the same density class of measures.

Proof. We have to show that for all positive functions $\varphi \in C\left(\partial \mathbb{H}^{n}\right)$ we have

$$
\int_{\partial \mathbb{H}^{n}} \varphi d \beta_{x}=0 \Longleftrightarrow \int_{\partial \mathbb{H}^{n}} \varphi d \beta_{y}=0
$$

This follows from the fact that $\mu_{x}$ and $\mu_{y}$ are in the same density class. Indeed, if $\Phi$ denotes the function $z \mapsto \int_{\partial \mathbb{H}^{n}} \varphi d \lambda_{z}$, since the developing measures are positive, $\Phi$ is positive, and

$$
\int_{\partial \mathbb{H}^{n}} \varphi d \beta_{x}=\int_{\partial \mathbb{H}^{n}} \Phi d \mu_{x}=0 \Longleftrightarrow 0=\int_{\partial \mathbb{H}^{n}} \Phi d \mu_{y}=\int_{\partial \mathbb{H}^{n}} \varphi d \beta_{y} .
$$


Lemma 3.3 For all $x \in \mathbb{H}^{k},\left\|\beta_{x}\right\|>0$.

Proof. It follows from condition (41) of Definition 2.10 and from the fact that $\left\|\mu_{x}\right\|>0$.

Corollary 3.4 For all $x \in \mathbb{H}^{k}$, the measure $\beta_{x}$ is not concentrated on two points.

Proof. By Lemma 3.3. $\beta_{x}$ is not the zero-measure. Suppose that $\beta_{x}$ has an atom of positive weight at $y_{0} \in \partial \mathbb{H}^{n}$. By Lemmas [3.1] and [3.2, $\beta_{x}$ has an atom of positive weight at each point of the $\rho(\Gamma)$-orbit of $y_{0}$, which contains most than two points because $\rho(\Gamma)$ is non-elementary.

It follows that for all $x \in \mathbb{H}^{k}$, the barycentre of the measure $\beta_{x}$ is welldefined and belongs to $\mathbb{H}^{n}$. We define the natural map $F: \mathbb{H}^{k} \rightarrow \mathbb{H}^{n}$ by

$$
F(x)=\operatorname{bar}\left(\beta_{x}\right) .
$$

By condition (11) of Lemma 2.8, the natural map is characterised by the implicit equation

$$
G(x, F(x))=0
$$

where

$$
\begin{aligned}
G(x, \xi) & =\int_{\partial \mathbb{H}^{n}} d B_{N(\xi, \theta)}(\cdot) d \beta_{x}(\theta) \\
& =\int_{\partial \mathbb{H}^{k}} \int_{\partial \mathbb{H}^{n}} d B_{N(\xi, \theta)}(\cdot) d \lambda_{z}(\theta) e^{-\delta(\Gamma) B_{K}(x, z)} d \mu_{O}(z) .
\end{aligned}
$$

The function $G$ is smooth because the Busemann functions $B_{K}$ and $B_{N}$ are smooth. Then, by the implicit function theorem, we get that $F$ is smooth. Moreover, by Lemma 3.1] and claim (2) of Lemma 2.8, it follows that $F$ is $\rho$-equivariant.

By differentiating equation (11) we get that for all $u \in T_{x} \mathbb{H}^{k}$ and $v \in$ $T_{F(x)} \mathbb{H}^{n}$

$$
\begin{aligned}
& \left.\int_{\partial \mathbb{H}^{k}} \int_{\partial \mathbb{H}^{n}} D d B_{N}\right|_{(F(x), \theta)}\left(d F_{x}(u), v\right) d \lambda_{z} d \mu_{x}= \\
= & \delta(\Gamma) \int_{\partial \mathbb{H}^{k}} \int_{\partial \mathbb{H}^{n}} d B_{N(F(x), \theta)}(v) d B_{K(x, z)}(u) d \lambda_{z} d \mu_{x} .
\end{aligned}
$$


Equation (2) is the analogous of equation (2.3) of [BCG99]. The proof of the properties of the natural map now goes exactly as in [BCG99, p. 152154], and the proof of Theorem [1.4 is complete.

\section{Weak extension of equivariant maps: exis- tence of developing measures}

In this section we prove Theorem [1.1. In particular, to obtain a family of developing measures, we show that any equivariant map weakly extend to the boundary in the setting of Borel measures. We keep here the notation fixed in Section 2, included the one of Theorem 2.6 for the Patterson-Sullivan measures.

The rough idea is the following. Consider the graphic $G(D)$ of $D$ as a subspace of $\overline{\mathbb{H}}^{k} \times \overline{\mathbb{H}}^{n}$. Then, for each measure $\mu$ on $\mathbb{H}^{k}$ we can consider the graphic measure $\eta$ on $\overline{\mathbb{H}}^{k} \times \overline{\mathbb{H}}^{n}$, that is, the only measure which is concentrated on $G(D)$ and whose push-forward $\pi_{K *} \eta$ is $\mu$. The sequence $\left\{\eta_{x}^{s}\right\}$ of the graphic measures corresponding to the measures $\left\{\mu_{x}^{s}\right\}$, weakly converges to a measure $\eta_{x}$ concentrated on $\partial \mathbb{H}^{k} \times \overline{\mathbb{H}}^{n}$. Then, by disintegrating the measure $\eta_{x}$ we obtain a family $\left\{\alpha_{z}\right\}_{z \in \partial \mathbb{H}^{k}}$ of measures on $\overline{\mathbb{H}}^{n}$. By making the convolution with the family of visual measures of $\partial \mathbb{H}^{n}$, we get a family of developing measures.

For each $x \in \mathbb{H}^{k}$ and $s>\delta(\Gamma)$ we define a positive Borel measure of finite mass on $\overline{\mathbb{H}}^{k} \times \overline{\mathbb{H}}^{n}$ as follows:

$$
\eta_{x}^{s}=\frac{1}{c(s)} \sum_{\gamma \in \Gamma} e^{-s d(x, \gamma O)} \delta_{(\gamma O, D(\gamma O))}=\frac{1}{c(s)} \sum_{\gamma \in \Gamma} e^{-s d(x, \gamma O)} \delta_{(\gamma O, \rho(\gamma) D(O))}
$$

where $\delta_{(x, y)}$ denotes the Dirac measure concentrated on $(x, y) \in \overline{\mathbb{H}}^{k} \times \overline{\mathbb{H}}^{n}$. The measures $\left\{\eta_{x}^{s}\right\}$ are the graphic measures associated to $\left\{\mu_{x}^{s}\right\}$ and $D$, and they are concentrated on $\mathbb{H}^{k} \times \mathbb{H}^{n}$. Note that $\eta_{x}^{s}=\mu_{x}^{s} \times\left\{\delta_{D(z)}\right\}$.

The group $\operatorname{Isom}\left(\mathbb{H}^{\mathrm{k}}\right) \times \operatorname{Isom}\left(\mathbb{H}^{\mathrm{n}}\right)$ acts on $\overline{\mathbb{H}}^{k} \times \overline{\mathbb{H}}^{n}$ by

$$
\left(g_{1}, g_{2}\right)(x, y)=\left(g_{1} x, g_{2} y\right) .
$$

Lemma 4.1 For all $s>\delta(\Gamma)$ the family $\left\{\eta_{x}^{s}\right\}$ is $\rho$-equivariant, that is, for all $x \in \mathbb{H}^{k}$ and $\psi \in \Gamma$

$$
\eta_{\psi x}^{s}=(\psi, \rho(\psi))_{*} \eta_{x}^{s}
$$


Proof.

$$
\begin{aligned}
\eta_{\psi x}^{s} & =\frac{1}{c(s)} \sum_{\gamma \in \Gamma} e^{-s d(\psi x, \gamma O)} \delta_{(\gamma O, D(\gamma O))}=\frac{1}{c(s)} \sum_{\gamma \in \Gamma} e^{-s d\left(x, \psi^{-1} \gamma O\right)} \delta_{(\gamma O, D(\gamma O))} \\
& =\frac{1}{c(s)} \sum_{\gamma \in \Gamma} e^{-s d(x, \gamma O)} \delta_{(\psi \gamma O, D(\psi \gamma O))}=\frac{1}{c(s)} \sum_{\gamma \in \Gamma} e^{-s d(x, \gamma O)} \delta_{(\psi \gamma O, \rho(\psi)(D(\gamma O)))} \\
& =(\psi, \rho(\psi))_{*} \eta_{x}^{s} .
\end{aligned}
$$

Now consider the family $\left\{\eta_{O}^{s}\right\}_{s>\delta(\Gamma)}$. Since $\left\|\eta_{O}^{s}\right\|=1$ for all $s$, there exists a sequence $s_{i} \rightarrow \delta(\Gamma)^{+}$such that $\eta_{O}^{s_{i}}$ weakly converges to a positive Borel measure $\eta_{O}$ of finite mass on $\overline{\mathbb{H}}^{n}$

$$
\eta_{O}^{s_{i}} \rightarrow \eta_{O}
$$

Moreover, by definition, for all $s>\delta(\Gamma)$ and $x \in \mathbb{H}^{k}$, the measure $\mu_{x}^{s}$ is the $\pi_{K}$-push-forward of $\eta_{x}^{s}$. Then, weak continuity of the push-forward implies

$$
\mu_{O}=\left(\pi_{K}\right)_{*} \eta_{O}
$$

By the theorem of disintegration of measures ([AFP00, Theorem 2.28], see also (DM78), for any positive Borel measure $\eta$ on $\overline{\mathbb{H}}^{k} \times \overline{\mathbb{H}}^{n}$, there exists a family of positive Borel measures $\left\{\alpha_{z}^{\eta}\right\}_{z \in \overline{\mathbb{H}}^{k}}$ such that, if $\mu=\left(\pi_{K}\right)_{*} \eta$, then $\eta=\mu \times\left\{\alpha_{z}^{\eta}\right\}$. Thus, for all $\varphi \in C\left(\overline{\mathbb{H}}^{k} \times \overline{\mathbb{H}}^{n}\right)$

$$
\iint_{\overline{\mathbb{H}}^{k} \times \overline{\mathbb{H}}^{n}} \varphi d \eta=\int_{\overline{\mathbb{H}}^{k}}\left(\int_{\overline{\mathbb{H}}^{n}} \varphi(x, y) d \alpha_{z}^{\eta}(y)\right) d \mu(z),
$$

and for $\mu$-almost $z$, the measure $\alpha_{z}^{\eta}$ is a probability measure. We say that the family $\left\{\alpha_{z}^{\eta}\right\}$ disintegrates the measure $\eta$ (compare disintegration with property (2) of Definition 2.10).

Moreover, the measures $\alpha_{z}^{\eta}$ 's are characterised by the following property. For all $z \in \overline{\mathbb{H}}^{k}$, let $\left\{U_{j}(z) \subset \overline{\mathbb{H}}^{k}\right\}_{j \in \mathbb{N}}$ be a sequence of nested small balls centred at $z$ such that $\cap_{j} U_{j}(z)=z$. For $j \in \mathbb{N}$, let $\psi_{j}^{z, \eta}: \overline{\mathbb{H}}^{k} \rightarrow \mathbb{R}$ be the following function

$$
\psi_{j}^{z, \eta}=\frac{\chi_{U_{j}(z)}}{\mu\left(U_{j}(z)\right)}
$$

where $\chi_{A}$ denotes the characteristic function of the set $A$ (note that $\psi_{j}^{z, \eta} \rightarrow \delta_{z}$ for $\mu$-almost $z)$. Then, for $\mu$-almost all $z$ and for all $\varphi \in C\left(\overline{\mathbb{H}}^{n}\right)$

$$
\int_{\overline{\mathbb{H}}^{n}} \varphi(y) d \alpha_{z}^{\eta}(y)=\lim _{j \rightarrow \infty} \iint_{\overline{\mathbb{H}}^{k} \times \overline{\mathbb{H}}^{n}} \psi_{j}^{z, \eta}(x) \varphi(y) d \eta(x, y) .
$$


From now on, the set $\left\{\alpha_{z}\right\}_{z \in \overline{\mathbb{H}}^{k}}$ will denote the family of measures that disintegrates $\eta_{O}$, where we set $\alpha_{z}=0$ for $z \in \mathbb{H}^{k}$.

Definition 4.2 For each $x \in \mathbb{H}^{k}$ we set $\eta_{x}=\mu_{x} \times\left\{\alpha_{z}\right\}$.

Theorem 4.3 For all $x \in \mathbb{H}^{k}$, the sequence $\left\{\eta_{x}^{s_{i}}\right\}$ weakly converges to $\eta_{x}$.

Proof. We fix $x \in \mathbb{H}^{k}$. Since the measures $\eta_{x}^{s_{i}}$ are bounded in norm, up to pass to subsequences, we can suppose that $\left\{\eta_{x}^{s_{i}}\right\}$ weakly converges to a measure $\widetilde{\eta}_{x}$. Let $\left\{\widetilde{\alpha}_{z}\right\}$ be the family that disintegrates $\widetilde{\eta}_{x}$. By weak continuity of push forward

$$
\pi_{K *}\left(\widetilde{\eta}_{x}\right)=\mu_{x}=\pi_{K *}\left(\eta_{x}\right) .
$$

Therefore, it is sufficient to show that $\widetilde{\alpha}_{z}=\alpha_{z}$ for $\mu_{O}$-almost all $z$. For any positive function $\varphi \in C\left(\overline{\mathbb{H}}^{n}\right)$ and $z \in \partial \mathbb{H}^{k}$

$$
\begin{aligned}
& \int_{\overline{\mathbb{H}}^{n}} \varphi(y) d \widetilde{\alpha}_{z}(y)=\lim _{j \rightarrow \infty} \iint_{\overline{\mathbb{H}}^{k} \times \overline{\mathbb{H}}^{n}} \psi_{j}^{z, \widetilde{\eta}_{x}}(\xi) \varphi(y) d \widetilde{\eta}_{x}(\xi, y) \\
= & \lim _{j \rightarrow \infty} \lim _{s_{i} \rightarrow \delta(\Gamma)^{+}} \iint_{\overline{\mathbb{H}}^{k} \times \overline{\mathbb{H}}^{n}} \psi_{j}^{z, \widetilde{\eta}_{x}}(\xi) \varphi(y) d \eta_{x}^{s_{i}}(\xi, y) \\
= & \lim _{j \rightarrow \infty} \lim _{s_{i} \rightarrow \delta(\Gamma)^{+}} \int_{\overline{\mathbb{H}}^{k}} \psi_{j}^{z, \widetilde{\eta}_{x}}(\xi) \varphi(D(\xi)) d \mu_{x}^{s_{i}}(\xi) \\
= & \lim _{j \rightarrow \infty} \lim _{s_{i} \rightarrow \delta(\Gamma)^{+}} \int_{U_{j}(z)} \varphi(D(\xi)) \frac{1}{\mu_{x}\left(U_{j}(z)\right)} d \mu_{x}^{s_{i}} \\
= & \lim _{j \rightarrow \infty} \lim _{s_{i} \rightarrow \delta(\Gamma)^{+}} \int_{U_{j}(z)} \varphi(D(\xi)) \frac{\mu_{O}\left(U_{j}(z)\right) e^{-\delta(\Gamma) B_{K}(x, z)}}{\mu_{x}\left(U_{j}(z)\right) \mu_{O}\left(U_{j}(z)\right) e^{-\delta(\Gamma) B_{K}(x, z)}} d \mu_{x}^{s_{i}}
\end{aligned}
$$

whence, using the definition of $\mu_{x}^{s_{i}}$, and setting

$$
A_{j}(z)=\frac{\mu_{O}\left(U_{j}(z)\right) e^{-\delta(\Gamma) B_{K}(x, z)}}{\mu_{x}\left(U_{j}(z)\right)},
$$

we get

$$
\begin{aligned}
& \int_{\overline{\mathbb{H}}^{n}} \varphi(y) d \widetilde{\alpha}_{z}(y) \\
= & \lim _{j \rightarrow \infty} A_{j}(z) \lim _{s_{i} \rightarrow \delta(\Gamma)^{+}} \int_{U_{j}(z)} \frac{\varphi(D(\xi))}{\mu_{O}\left(U_{j}(z)\right) e^{-\delta(\Gamma) B_{K}(x, z)}} d \mu_{x}^{s_{i}}(\xi) \\
= & \lim _{j \rightarrow \infty} A_{j}(z) \lim _{s_{i} \rightarrow \delta(\Gamma)^{+}} \frac{1}{c\left(s_{i}\right)} \sum_{\substack{g \in \Gamma \\
g O \in U_{j}}} \frac{\varphi(D(g O)) e^{-s_{i} d(x, g O)}}{\mu_{O}\left(U_{j}(z)\right) e^{-\delta(\Gamma) B_{K}(x, z)}} .
\end{aligned}
$$


Moreover,

$$
\begin{aligned}
& \frac{\varphi(D(g O)) e^{-s_{i} d(x, g O)}}{\mu_{O}\left(U_{j}(z)\right) e^{-\delta(\Gamma) B_{K}(x, z)}} \\
= & \frac{\varphi(D(g O))}{\mu_{O}\left(U_{j}(z)\right)} \cdot \frac{e^{-s_{i}(d(x, g O)-d(O, g O))}}{e^{-\delta(\Gamma) B_{K}(x, z)}} \cdot e^{-s_{i} d(O, g O)} \\
= & \frac{\varphi(D(g O))}{\mu_{O}\left(U_{j}(z)\right)} \cdot \frac{e^{-\delta(\Gamma)(d(x, g O)-d(O, g O))}}{e^{-\delta(\Gamma) B_{K}(x, z)}} \cdot \frac{e^{-s_{i}(d(x, g O)-d(O, g O))}}{e^{-\delta(\Gamma)(d(x, g O)-d(O, g O))}} \cdot e^{-s_{i} d(O, g O)} .
\end{aligned}
$$

From the definition of the Busemann function $B_{K}$, it follows that for all $z \in \partial \mathbb{H}^{k}$ and $p \in \mathbb{H}^{k}$

$$
\lim _{\xi \rightarrow z}(d(p, \xi)-d(O, \xi))=B_{K}(p, z)
$$

Therefore, there exist two sequences $\left\{E_{j}^{+}\right\}$and $\left\{E_{j}^{-}\right\}$, converging to 1 as $j \rightarrow \infty$, and such that for all $g \in \Gamma$ with $g O \in U_{j}(z)$

$$
E_{j}^{-} \leq \frac{e^{-\delta(\Gamma)(d(x, g O)-d(O, g O))}}{e^{-\delta(\Gamma) B_{K}(x, z)}} \leq E_{j}^{+} .
$$

Moreover, equation (6) implies that the term $(d(x, g O)-d(O, g O))$ is bounded, so for each $j$

$$
\lim _{s_{i} \rightarrow \delta(\Gamma)^{+}} \frac{e^{-s_{i}(d(x, g O)-d(O, g O))}}{e^{-\delta(\Gamma)(d(x, g O)-d(O, g O))}}=1
$$

uniformly on $U_{j}$. Whence, since $\varphi$ is positive,

$$
\begin{aligned}
& \lim _{j \rightarrow \infty} A_{j}(z) \lim _{s_{i} \rightarrow \delta(\Gamma)^{+}} \frac{1}{c\left(s_{i}\right)} \sum_{\substack{g \in \Gamma \\
g O \in U_{j}}} \frac{\varphi(D(g O)) e^{-s_{i} d(x, g O)}}{\mu_{O}\left(U_{j}(z)\right) e^{-\delta(\Gamma) B_{K}(x, z)}} \\
\leq & \lim _{j \rightarrow \infty} A_{j}(z) E_{j}^{+} \lim _{s_{i} \rightarrow \delta(\Gamma)^{+}} \frac{1}{c\left(s_{i}\right)} \sum_{\substack{g \in \Gamma \\
g O \in U_{j}}} \frac{\varphi(D(g O))}{\mu_{O}\left(U_{j}(z)\right)} \cdot e^{-s_{i} d(O, g O)} \\
= & \lim _{j \rightarrow \infty} A_{j}(z) E_{j}^{+} \lim _{s_{i} \rightarrow \delta(\Gamma)^{+}} \int_{U_{j}(z)} \frac{\varphi(D(x))}{\mu_{O}\left(U_{j}(z)\right)} d \mu_{O}^{s_{i}}(x)
\end{aligned}
$$

and similarly for $E_{j}^{-}$. Since for $\mu_{O}$-almost all $z$ we have $\lim _{j} A_{j}(z)=1$, and 
since $E_{j}^{ \pm} \rightarrow 1$, we get that $\mu_{O}$-almost everywhere

$$
\begin{aligned}
& \lim _{j \rightarrow \infty} A_{j}(z) E_{j}^{ \pm} \lim _{s_{i} \rightarrow \delta(\Gamma)^{+}} \int_{U_{j}(z)} \frac{\varphi(D(\xi))}{\mu_{O}\left(U_{j}(z)\right)} d \mu_{O}^{s_{i}}(\xi) \\
= & \lim _{j \rightarrow \infty} \lim _{s_{i} \rightarrow \delta(\Gamma)^{+}} \int_{U_{j}(z)} \varphi(D(\xi)) \frac{1}{\mu_{O}\left(U_{j}(z)\right)} d \mu_{O}^{s_{i}} \\
= & \lim _{j \rightarrow \infty} \lim _{s_{i} \rightarrow \delta(\Gamma)^{+}} \int_{\overline{\mathbb{H}}^{k}} \psi_{j}^{z, \eta_{O}}(\xi) \varphi(D(\xi)) d \mu_{O}^{s_{i}}(\xi) \\
= & \lim _{j \rightarrow \infty} \lim _{s_{i} \rightarrow \delta(\Gamma)^{+}} \iint_{\overline{\mathbb{H}}^{k} \times \overline{\mathbb{H}}^{n}} \psi_{j}^{z, \eta_{O}}(\xi) \varphi(y) d \eta_{O}^{s_{i}}(\xi, y) \\
= & \lim _{j \rightarrow \infty} \iint_{\overline{\mathbb{H}}^{k} \times \overline{\mathbb{H}}^{n}} \psi_{j}^{z, \eta_{O}}(\xi) \varphi(y) d \eta_{O}(\xi, y) \\
= & \int_{\overline{\mathbb{H}}^{n}} \varphi(y) d \alpha_{z}(y) .
\end{aligned}
$$

Finally, from equations (31) - (15),$\mu_{O}$-almost everywhere we get

$$
\int_{\overline{\mathbb{H}}^{n}} \varphi(y) d \alpha_{z}(y) \leq \int_{\overline{\mathbb{H}}^{n}} \varphi(y) d \widetilde{\alpha}_{z}(y) \leq \int_{\overline{\mathbb{H}}^{n}} \varphi(y) d \alpha_{z}(y)
$$

and the claim follows.

In particular, Theorem4.3 implies that the measures $\eta_{x}$ 's are $\rho$-equivariant. Indeed, since the measures $\left\{\eta_{x}^{s}\right\}$ are $\rho$-equivariant, and since the push-forward is continuous for the weak convergence, for all $x \in \mathbb{H}^{k}$ and $\gamma \in \Gamma$

$$
\eta_{\gamma x}^{s_{i}}=(\gamma, \rho(\gamma))_{*} \eta_{x}^{s_{i}} \rightarrow(\gamma, \rho(\gamma))_{*} \eta_{x}
$$

Moreover, since $\pi_{K *}\left(\eta_{x}\right)=\mu_{x}$, each measure $\eta_{x}$ is concentrated in $\partial \mathbb{H}^{k} \times \overline{\mathbb{H}}^{n}$.

Lemma 4.4 The family $\left\{\alpha_{z}\right\}$ is $\rho$-equivariant, that is, for all $\gamma \in \Gamma$ and $\mu_{O}$-almost all $z \in \partial \mathbb{H}^{k}$

$$
\alpha_{\gamma z}=\rho(\gamma)_{*} \alpha_{z}
$$

Proof. From point (3) of Theorems 2.6 and the the $\rho$-equivariance of the $\eta_{x}$ 's, 
it follows that for all $\varphi \in C\left(\overline{\mathbb{H}}^{k} \times \overline{\mathbb{H}}^{n}\right)$

$$
\begin{aligned}
& \iint_{\partial \mathbb{H}^{k} \times \overline{\mathbb{H}}^{n}} \varphi(\gamma z, y) d \alpha_{\gamma z}(y) d \mu_{O}(z) \\
= & \iint_{\partial \mathbb{H}^{k} \times \overline{\mathbb{H}}^{n}} \varphi(z, y) d \alpha_{z}(y) d \mu_{\gamma O}(z) \\
= & \iint_{\overline{\mathbb{H}}^{k} \times \overline{\mathbb{H}}^{n}} \varphi(z, y) d \eta_{\gamma O}=\iint_{\overline{\mathbb{H}}^{k} \times \overline{\mathbb{H}}^{n}} \varphi(\gamma z, \rho(\gamma) y) d \eta_{O} \\
= & \iint_{\partial \mathbb{H}^{k} \times \overline{\mathbb{H}}^{n}} \varphi(\gamma z, \rho(\gamma) y) d \alpha_{z}(y) d \mu_{O}(z) \\
= & \iint_{\overline{\mathbb{H}}^{k} \times \overline{\mathbb{H}}^{n}} \varphi(\gamma z, y) d\left(\rho(\gamma)_{*} \alpha_{z}\right)(y) d \mu_{O}(z) .
\end{aligned}
$$

Whence, the measures $\alpha_{\gamma z}$ and $\rho(\gamma)_{*} \alpha_{z}$ equal $\mu_{O}$-almost everywhere.

Now, for each $y \in \overline{\mathbb{H}}^{n}$ let $\nu_{y}$ be the visual measure on $\partial \mathbb{H}^{n}$ centred at $y$. More precisely, choose the disc model of $\mathbb{H}^{n}$ whose centre is $O$ and let $\nu_{O}$ be the standard probability measure on $S^{n-1} \simeq \partial \mathbb{H}^{n}$. Then, for all $g \in \operatorname{Isom}\left(\mathbb{H}^{\mathrm{n}}\right)$ define

$$
\nu_{g O}=g_{*} \nu_{O}
$$

This definition is not ambiguous because $\nu_{O}$ is $\operatorname{Stab}(O)$-invariant, where $\operatorname{Stab}(O)=\left\{g \in \operatorname{Isom}\left(\mathbb{H}^{\mathrm{n}}\right): g(O)=O\right\}$. For $y \in \partial \mathbb{H}^{n}$ simply define $\nu_{y}=\delta_{y}$.

For all $z \in \partial \mathbb{H}^{k}$ define $\lambda_{z}$ a measure on $\partial \mathbb{H}^{n}$ by $\lambda_{z}=\alpha_{z} *\left\{\nu_{y}\right\}$. That is, for all $\varphi \in C\left(\partial \mathbb{H}^{n}\right)$

$$
\int_{\partial \mathbb{H}^{n}} \varphi d \lambda_{z}=\int_{\overline{\mathbb{H}}^{n}}\left(\int_{\partial \mathbb{H}^{n}} \varphi(\theta) d \nu_{y}(\theta)\right) d \alpha_{z}(y) .
$$

Note that such an integral is well-defined because, for any $\varphi$, the function $y \mapsto \int_{\partial \mathbb{H}^{n}} \varphi d \nu_{y}$ is continuous in $y$.

Remark 4.5 Since the measure $\nu_{y}$ depends continuously on y, by Lemma[2.11, the convolution with the family of visual measures is weakly continuous.

We show now that $\left\{\lambda_{z}\right\}_{z \in \partial \mathbb{H}^{k}}$ is a family of developing measures for $\rho$.

Lemma 4.6 The family $\left\{\lambda_{z}\right\}$ is $\rho$-equivariant, that is, for $\mu_{O}$-almost all $z \in \partial \mathbb{H}^{k}$ and all $\gamma \in \Gamma$, we have $\lambda_{\gamma z}=\rho(\gamma)_{*} \lambda_{z}$. 
Proof. By Lemma 4.4, for $\mu_{O}$-almost all $z \in \partial \mathbb{H}^{k}$ and all $\varphi \in C\left(\partial \mathbb{H}^{n}\right)$

$$
\begin{aligned}
\int_{\partial \mathbb{H}^{n}} \varphi d \lambda_{\gamma z} & =\int_{\overline{\mathbb{H}}^{n}} \int_{\partial \mathbb{H}^{n}} \varphi(\theta) d \nu_{y}(\theta) d \alpha_{\gamma z}(y)=\int_{\mathbb{\mathbb { H }}^{n}} \int_{\partial \mathbb{H}^{n}} \varphi(\theta) d \nu_{\rho(\gamma) y}(\theta) d \alpha_{z}(y) \\
& =\int_{\overline{\mathbb{H}}^{n}} \int_{\partial \mathbb{H}^{n}} \varphi(\rho(\gamma) \theta) d \nu_{y}(\theta) d \alpha_{z}(y) \\
& =\int_{\partial \mathbb{H}^{n}} \varphi \circ \rho(\gamma) d \lambda_{z}=\int_{\partial \mathbb{H}^{n}} \varphi d\left(\rho(\gamma)_{*} \lambda_{z}\right) .
\end{aligned}
$$

Lemma 4.7 For any $\varphi \in C\left(\partial \mathbb{H}^{n}\right)$, the function $z \mapsto \int_{\partial \mathbb{H}^{n}} \varphi(\theta) d \lambda_{z}(\theta)$ is $\mu_{O}$-integrable.

Proof. This follows directly from the definition of $\lambda_{z}$, because the family $\left\{\alpha_{z}\right\}$ disintegrates $\eta_{O}$, and $\mu_{O}=\pi_{K *} \eta_{O}$.

Lemma 4.8 For $\mu_{O}$-almost all $z \in \partial \mathbb{H}^{k}$, we have $\left\|\lambda_{z}\right\|=1$.

Proof. For all $x \in \mathbb{H}^{k}$ and $y \in \partial \mathbb{H}^{n}$, the measures $\eta_{x}$ and $\nu_{y}$ are positive. Then the measures $\alpha_{z}$ 's are positive, and this implies that the measures $\lambda_{z}$ 's are positive. Thus

$$
\left\|\lambda_{z}\right\|=\int_{\partial \mathbb{H}^{n}} 1 d \lambda_{z}=\int_{\overline{\mathbb{H}}^{n}} \int_{\partial \mathbb{H}^{n}} 1 d \nu_{y}(\theta) d \alpha_{z}(y)=\int_{\overline{\mathbb{H}}^{n}} 1 d \alpha_{z}=\left\|\alpha_{z}\right\|=1
$$

$\mu_{O}$-almost everywhere because $\left\{\alpha_{z}\right\}$ disintegrates $\eta_{O}$.

Therefore, the family $\left\{\lambda_{z}\right\}$ satisfies properties (11) - (4) of Definition 2.10. So it is a family of developing measure for $\rho$, and the proof of Theorem 1.1 is complete.

\section{$5 \quad$ Sequence of $\varepsilon$-natural maps}

In this section we show that the natural map constructed in Sections 3 and 4 is the limit of a sequence of $\varepsilon$-natural maps. We keep the notation of Sections 2 4. Through this section we suppose that $\Gamma$ diverges at $\delta(\Gamma)$; by Lemma 2.5 , this is the case if $\mathbb{H}^{k} / \Gamma$ has finite volume. 
Let $A \subset \mathbb{H}^{k}$ be the Dirichlet domain of $O$. The set $A$ is a fundamental domain for $\Gamma$ containing $O$. Let $\sigma$ be any Borel probability measure on $A$.

Definition 5.1 For each $s>\delta(\Gamma)$ and $x \in \mathbb{H}^{k}$ we define $m_{x}^{s}$ a positive Borel measure on $\mathbb{H}^{k}$ by

$$
m_{x}^{s}=\frac{1}{c(s)} \sum_{\gamma \in \Gamma} e^{-s d(x, \gamma O)} \gamma_{*} \sigma
$$

Note that if $\sigma=\delta_{O}$, then $m_{x}^{s}=\mu_{x}^{s}$.

Lemma 5.2 Let $\left\{\gamma_{n}\right\}$ be any sequence of elements of $\Gamma$. If $\gamma_{n}(O)$ converges to a point $\theta \in \partial \mathbb{H}^{k}$, then for any sequence $\left\{x_{n}\right\} \in A$, the sequence $\left\{\gamma_{n}\left(x_{n}\right)\right\}$ converges to $\theta$.

Proof. Suppose the contrary. Since $\overline{\mathbb{H}}^{k}$ is compact, up to passing to a subsequence, we can suppose that $\left\{\gamma_{n}\left(x_{n}\right)\right\}$ converges to a point $\zeta \neq \theta$ in $\overline{\mathbb{H}}^{k}$. Then, the geodesics joining $\gamma_{n}\left(x_{n}\right)$ to $\gamma_{n}(O)$ accumulate near the geodesic between $\zeta$ and $\theta$. This can not happen because $\Gamma$ is discrete.

Theorem 5.3 For each $x \in \mathbb{H}^{k}$, if $\mu_{x}$ denotes the Patterson-Sullivan measure constructed as in Theorem [2.6, then

$$
m_{x}^{s} \rightarrow \mu_{x}
$$

in $\overline{\mathbb{H}}^{k}$, when $s \rightarrow \delta(\Gamma)^{+}$.

Proof. We have to show that for each $\varphi \in C\left(\overline{\mathbb{H}}^{k}\right), \int \varphi d m_{x}^{s} \rightarrow \int \varphi d \mu_{x}$. Let $\varphi \in C\left(\overline{\mathbb{H}}^{k}\right)$. Since $\mu_{x}^{s} \rightarrow \mu_{x}$, we will have finished by proving that

$$
\lim _{s \rightarrow \delta(\Gamma)^{+}}\left|\int_{\overline{\mathbb{H}}^{k}} \varphi d m_{x}^{s}-\int_{\overline{\mathbb{H}}^{k}} \varphi \mu_{x}^{s}\right|=0 .
$$

Let $C>0$ be a small constant and let $A_{1} \subset A$ be a compact set such that $O \in A_{1}$ and $\sigma\left(A \backslash A_{1}\right)<C$. Since the supports of the measures $m_{x}^{s}$ and $\mu_{x}^{s}$ are contained in $\mathbb{H}^{k}$, and since $O \in A$, we have

$$
\int_{\overline{\mathbb{H}}^{k}} \varphi d\left(m_{x}^{s}-\mu_{x}^{s}\right)=\sum_{\gamma \in \Gamma} \int_{\gamma\left(A_{1}\right)} \varphi d\left(m_{x}^{s}-\mu_{x}^{s}\right)+\sum_{\gamma \in \Gamma} \int_{\gamma\left(A \backslash A_{1}\right)} \varphi d m_{x}^{s} .
$$


Looking at the second summand,

$$
\begin{aligned}
& \left|\sum_{\gamma \in \Gamma} \int_{\gamma\left(A \backslash A_{1}\right)} \varphi d m_{x}^{s}\right| \leq \max (|\varphi|) \frac{1}{c(s)} \sum_{\gamma \in \Gamma} \int_{\gamma\left(A \backslash A_{1}\right)} e^{-s d(x, \gamma O)} d \gamma_{*} \sigma(\xi) \\
& =\max (|\varphi|) \frac{1}{c(s)} \sum_{\gamma \in \Gamma} e^{-s d(x, \gamma O)} \sigma\left(A \backslash A_{1}\right) \leq C \cdot \max (\varphi) \cdot\left\|\mu_{x}^{s}\right\| .
\end{aligned}
$$

We estimate now the first summand. Since $c(s) \rightarrow \infty$, for any finite subset $\Gamma_{1}$ of $\Gamma$

$$
\lim _{s \rightarrow \delta(\Gamma)^{+}}\left|\sum_{\gamma \in \Gamma_{1}} \int_{\gamma\left(A_{1}\right)} \varphi d m_{x}^{s}\right|=\lim _{s \rightarrow \delta(\Gamma)^{+}}\left|\sum_{\gamma \in \Gamma_{1}} \int_{\gamma\left(A_{1}\right)} \varphi d \mu_{x}^{s}\right|=0 .
$$

Moreover, since $\varphi$ is continuous, it is uniformly continuous on $\overline{\mathbb{H}}^{k}$ for any metric that induces the usual topology on $\overline{\mathbb{H}}^{k}$ (recall that the hyperbolic metric of $\mathbb{H}^{k}$ is not a metric on $\overline{\mathbb{H}}^{k}$ ). Therefore, Lemma 5.2 implies that, except that for a finite number of elements of $\Gamma$, we have

$$
|\varphi(\gamma O)-\varphi(\gamma \xi)|<C
$$

independently on $\gamma$ and on $\xi \in A_{1}$. Let $\Gamma_{1}$ be a huge finite subset of $\Gamma$ such that (20) holds for $\gamma \in \Gamma \backslash \Gamma_{1}$. Then,

$$
\begin{aligned}
& \left|\sum_{\gamma \in \Gamma \backslash \Gamma_{1}} \int_{\gamma\left(A_{1}\right)} \varphi d\left(m_{x}^{s}-\mu_{x}^{s}\right)\right| \\
= & \left|\frac{1}{c(s)} \sum_{\gamma \in \Gamma \backslash \Gamma_{1}} e^{-s d(x, \gamma O)}\left(\int_{\gamma\left(A_{1}\right)} \varphi(\xi) d \gamma_{*} \sigma(\xi)-\varphi(\gamma O)\left(\sigma\left(A_{1}\right)+\sigma\left(A \backslash A_{1}\right)\right)\right)\right| \\
\leq & C \int_{\overline{\mathbb{H}}^{k}}|\varphi| d \mu_{x}^{s}+\frac{1}{c(s)} \sum_{\gamma \in \Gamma \backslash \Gamma_{1}} e^{-s d(x, \gamma O)} \int_{A_{1}}|\varphi(\gamma \xi)-\varphi(\gamma O)| d \sigma(\xi) \\
\leq & C \int_{\overline{\mathbb{H}}^{k}}|\varphi| d \mu_{x}^{s}+\frac{C}{c(s)} \sum_{\gamma \in \Gamma \backslash \Gamma_{1}} e^{-s d(x, \gamma O)} \cdot \sigma\left(A_{1}\right) \leq C \int_{\overline{\mathbb{H}}^{k}}(1+|\varphi|) d \mu_{x}^{s} .
\end{aligned}
$$

Whence the claim follows, combining with (16), (18) and (19), since $C$ can be chosen arbitrarily small.

Now we proceed as in Sections 3 and 4 . Namely, we fix a $\rho$-equivariant map $D$, we define measures $n_{x}^{s}=m_{x}^{s} \times\left\{\delta_{D(z)}\right\}$, and we chose a sequence $s_{i} \rightarrow \delta(\Gamma)^{+}$such that $n_{O}^{s_{i}}$ converges to a measure $n_{O}$. We disintegrate $n_{O}$ as 
$n_{O}=\mu_{O} \times\left\{a_{z}\right\}$, and we define $n_{x}=\mu_{x} \times\left\{a_{z}\right\}$. As in Theorem 4.3 one can show that $n_{x}^{s_{i}} \rightarrow n_{x}$. We define then $b_{x}^{s}=\left(D_{*} m_{x}^{s}\right) *\left\{\nu_{y}\right\}=\pi_{N *}\left(n_{x}^{s}\right) *\left\{\nu_{f}\right\}$, and $b_{x}=\pi_{N *}\left(n_{x}\right) *\left\{\nu_{f}\right\}$.

Finally, for each $s>\delta(\Gamma)$ we set $s=(1+\varepsilon) \delta(\Gamma)$ and we define maps $F(x)=\operatorname{bar}\left(b_{x}\right)$ and $F_{\varepsilon}(x)=\operatorname{bar}\left(b_{x}^{s}\right)$. The map $F$ is a natural map, that is, it has the properties claimed in Theorem 1.2. The maps $F_{\varepsilon}$ the $\varepsilon$-natural maps constructed in [BCG99], so they are smooth, $\rho$-equivariant, and for all $p \geq 3$ and $\varepsilon>0, \operatorname{Jac}_{p} F_{\varepsilon}(x) \leq\left(\frac{(1+\varepsilon) \delta(\Gamma)}{p-1}\right)^{p}$.

Proposition 5.4 The maps $F_{\varepsilon_{i}}$ punctually converge to the map $F$.

Proof. From the weak continuity of the push-forward and from Lemma 2.11, we get

$$
b_{x}^{s_{i}} \rightarrow b_{x} .
$$

Then, the claim follows from Point 3 of Lemma 2.8.

Remark 5.5 Arguing as in Proposition 5.4, it can be shown that the derivatives of $F_{\varepsilon_{i}}$ converges the ones of $F$.

\section{Measurable extension of natural maps}

This section is devoted to prove Theorem 1.7. We keep here the notation of previous sections. In particular we recall that $\left\{\mu_{x}\right\}_{x \in \mathbb{H}^{k}}$ is the family of Patterson-Sullivan measures, and that $\left\{\lambda_{z}\right\}_{z \in \partial \mathbb{H}^{k}}$ is a family of developing measures.

Definition 6.1 Let $\left\{\gamma_{i} O\right\}$ be a sequence in the $\Gamma$-orbit of $O$. We say that $\gamma_{i} O$ conically converges to $\omega \in \partial \mathbb{H}^{k}$ if $\gamma_{i} O \rightarrow \omega$ and there exists a geodesic $\sigma$, ending at $\omega$, such that the distance of $\gamma_{i} O$ from $\sigma$ is bounded. The conical limit set of $\Gamma$, denoted by $\Lambda_{c}$, is the set of the limits of conically converging sequences in the $\Gamma$-orbit of $O$.

Clearly, the conical limit set is a sub-set of the limit set of $\Gamma$. In order to prove that the natural maps extend to the boundary we need the following result. 
Theorem 6.2 For each $f \in L^{1}\left(\partial \mathbb{H}^{k}, \mu_{O}\right)$ there exists a set $Z$ with $\mu_{O}(Z)=0$ such that for all $\omega \in \Lambda_{c} \backslash Z$, and for any sequence $\left\{\gamma_{i}\right\} \subset \Gamma$ such that $\gamma_{i} O$ conically converges to $\omega$

$$
\int_{\partial \mathbb{H}^{k}} f(\theta) d \mu_{\gamma_{i} O}(\theta) \rightarrow f(\omega) .
$$

Before proving Theorem 6.2 we show how it implies Theorem 1.7 Proof of Theorem 1.7. By definition (see Section 2) we have

$$
\begin{aligned}
& \mathcal{B}_{\beta_{x}}(y)=\int_{\partial \mathbb{H}^{n}} B_{N}(y, \theta) d \beta_{x}(\theta)=\int_{\partial \mathbb{H}^{k}} \int_{\partial \mathbb{H}^{n}} B_{N}(y, \theta) d \lambda_{z}(\theta) d \mu_{x}(z) \\
= & \int_{\partial \mathbb{H}^{k}} \mathcal{B}_{\lambda_{z}}(y) d \mu_{x}(z)
\end{aligned}
$$

and a similar formula holds for the derivatives of $\mathcal{B}_{\beta_{x}}$. Therefore, for each $y$ there exists a $\mu_{O}$-negligible set $Z \subset \partial \mathbb{H}^{k}$ such that for all $\omega \in \partial \mathbb{H}^{k} \backslash Z$ we have

$$
\lim _{x_{i} \rightarrow \omega} \mathcal{B}_{\beta_{x}}(y)=B_{\lambda_{\omega}}(y)
$$

Where " $\lim _{x \rightarrow \omega}$ " means "for any sequence $\left\{x_{i}\right\}$ in the $\Gamma$-orbit of $O$, conically converging to $\omega . . . "$. The same statement holds for the derivatives of $\mathcal{B}_{\beta_{x}}$. Now, let $Y$ be a countable dense subset of $\mathbb{H}^{n}$. Then, there exists a $\mu_{O^{-}}$ negligible set $W \subset \partial \mathbb{H}^{k}$ such that the above limit holds for all $\omega \in \partial \mathbb{H}^{k} \backslash W$, all $y \in Y$ and all the derivatives of $\mathcal{B}_{\beta_{x}}$. It follows that the barycentre of $\beta_{x}$, that is the unique point of minimum of $\mathcal{B}_{\beta_{x}}$, converges to the barycentre of $\lambda_{\omega}$, which is well-defined because $\lambda_{\omega}$ is not the sum of two Dirac deltas with equal weights. Therefore, if $F$ denotes the natural map constructed using the family $\left\{\lambda_{z}\right\}$, setting $\bar{F}(\omega)=\operatorname{bar}\left(\lambda_{\omega}\right)$, we have that for $\mu_{O}$-almost all $\omega$, for any sequence $\left\{\gamma_{i} O\right\}$ conically converging to $\omega$

$$
\lim _{\gamma_{i} O \rightarrow \omega} F\left(\gamma_{i} O\right)=\bar{F}(\omega) .
$$

The map $\bar{F}$ is measurable because it can be viewed as a limit of continuous functions. Finally, it is readily checked that $F\left(\gamma_{i} O\right)$ and $F\left(\gamma_{i} x\right)$ have the same limit. This completes the proof of Theorem 1.7.

Proof of Theorem 6.2 The map $x \mapsto \int_{\partial \mathbb{H}^{k}} f(\theta) d \mu_{x}(\theta)$ can be viewed as the harmonic extension of $f$, and one can prove its convergence to $f$ along cones and almost everywhere by using standard techniques of harmonic analysis. We give a proof for completeness. 
For the whole proof, we work in the half space model $\mathbb{H}^{k}=\mathbb{R}^{k-1} \times \mathbb{R}^{+}$, using the following notation. For a point $x \in \mathbb{H}^{k}$, we denote by $\left(x^{\prime}, x_{k}\right) \in$ $\mathbb{R}^{k-1} \times \mathbb{R}^{+}$its coordinates in the half-space model, by the symbol $|x|$ we denote the Euclidean norm of $x$ in the model, and $B(\omega, r)$ will denote the ball of center $\omega$ and Euclidean radius $r$. In the half-space model, setting $\delta=\delta(\Gamma)$ we have

$$
e^{-\delta B^{K}(x, \xi)}=\left(\frac{x_{k}\left(1+|\xi|^{2}\right)}{\left|\xi-x^{\prime}\right|^{2}+x_{k}^{2}}\right)^{\delta}
$$

and for all $\gamma \in \Gamma$, if $x=\gamma O$

$$
1=\left\|\mu_{O}\right\|=\left\|\gamma_{*} \mu_{O}\right\|=\left\|\mu_{\gamma O}\right\|=\int_{\partial \mathbb{H}^{k}}\left(\frac{x_{k}\left(1+|\xi|^{2}\right)}{\left|\xi-x^{\prime}\right|^{2}+x_{k}^{2}}\right)^{\delta} .
$$

We will work on a fixed ball of center 0 and radius $R$ of $\mathbb{R}^{k-1}$. This is not restrictive because proving convergence almost everywhere for all balls is equivalent to prove convergence almost everywhere.

For all $\omega \in \mathbb{R}^{k-1}$, we denote by $C_{\omega}(\alpha)$ the vertical cone in $\mathbb{H}^{k-1} \times \mathbb{R}^{+}$of vertex $\omega$ and emi-angle $\alpha$. For any non-negative $g \in L^{1}\left(\partial \mathbb{H}^{k}, \mu_{O}\right), \omega \in \partial \mathbb{H}^{k}$, $\alpha \in(0, \pi / 2)$ we define the maximal operator $M_{\alpha} g(\omega)$ by

$$
M_{\alpha} g(\omega)=\sup _{\gamma \in \Gamma, \gamma O \in C_{\omega}(\alpha)} \int_{\partial \mathbb{H}^{k}} g(\xi) d \mu_{\gamma O}(\xi)
$$

and the so-called Hardy Littlewood operator $N g(\omega)$ by

$$
N g(\omega)=\sup _{r>0} \frac{1}{\mu_{O}(B(\omega, r))} \int_{B(\omega, r)} g(\xi) d \mu_{O}(\xi) .
$$

From now on, the symbol $c$ will denote a generic constant, and different occurrences may denote different constants. If not specified, the constants do not depend on the other quantities we are considering.

Lemma 6.3 There exists a constant $c$ such that for every point $\omega$ of the limit set of $\Gamma$ and any $r>0$

$$
\mu_{O}(B(\omega, r)) \leq c r^{\delta}
$$

Proof. For all $x \in \mathbb{H}^{k}$

$$
\left\|\mu_{x}\right\|=\int_{\partial \mathbb{H}^{k}}\left(\frac{x_{k}\left(1+|\xi|^{2}\right)}{\left|\xi-x^{\prime}\right|^{2}+x_{k}^{2}}\right)^{\delta} \geq \int_{\partial \mathbb{H}^{k}}\left(\frac{x_{k}}{\left|\xi-x^{\prime}\right|^{2}+x_{k}^{2}}\right)^{\delta} .
$$


Suppose now that $x$ is of the form $x=\gamma O$, with

$$
c_{1} r \leq|x-\omega| \leq c_{2} r
$$

Then $\left|\xi-x^{\prime}\right| \leq|\xi-\omega|+\left|x^{\prime}-\omega\right| \leq\left(1+c_{2}\right) r$ for any $\xi \in B(\omega, r)$. Then

$$
1=\left\|\mu_{x}\right\|=\int_{\partial \mathbb{H}^{k}}\left(\frac{x_{k}}{\left|\xi-x^{\prime}\right|^{2}+x_{k}^{2}}\right)^{\delta} \geq c \frac{1}{r^{\delta}} \mu_{O}(B(\omega, r))
$$

and the claim holds for such points. Let $a>1$ and for $j \in \mathbb{Z}$, consider the set $A_{j}=\left\{x \in \overline{\mathbb{H}}^{k}:|x-\omega| \in\left[a^{j}, a^{j+1}\right)\right\}$. If for a certain $j \in \mathbb{Z}$ the set $A_{j}$ contains a point of the $\Gamma$-orbit of $O$, then for $r \in\left[a^{j}, a^{j+1}\right]$

$$
\frac{r}{a} \leq \frac{a^{j+1}}{a}=a^{j} \leq x \leq a^{j+1}=a^{j} a \leq r a
$$

and inequalities (21) hold with $c_{2}=a=1 / c_{1}$. Let now $r>0$. Since $\omega$ lies on the limit set, there exists $j$ with $a^{j} \leq r$ and such that $A_{j}$ intersects the $\Gamma$-orbit of $O$. Let $j_{0}$ be the maximum of such $j$ 's. If $a^{j_{0}+1} \geq r$, we have finished. Otherwise, since $\mu_{O}$ is concentrated on the limit set

$$
\mu_{0}(B(\omega, r))=\mu\left(B\left(\omega, a^{j_{0}+1}\right)\right) \leq c\left(a^{j_{0}+1}\right)^{\delta} \leq c r^{\delta}
$$

This completes the proof of Lemma 6.3

Lemma 6.4 There exists a constant $c$, depending only on $\alpha$, such that for any non-negative $g \in L^{1}\left(\partial \mathbb{H}^{k}, \mu_{O}\right), \omega \in \partial \mathbb{H}^{k}, \alpha \in(0, \pi / 2)$, we have

$$
M_{\alpha} g(\omega) \leq c N g(\omega)
$$


Proof. Let $x=\gamma O$ with $\gamma \in \Gamma$ and $\gamma O \in C_{\omega}(\alpha)$. As noticed above, it is not restrictive to work in the ball $B(0, R)$.

$$
\begin{aligned}
& \int_{\partial \mathbb{H}^{k}} g(\xi) d \mu_{x}(\xi) \leq(1+R)^{\delta} \int_{\partial \mathbb{H}^{k}} g(\xi)\left(\frac{x_{k}}{\left|\xi-x^{\prime}\right|^{2}+x_{k}^{2}}\right)^{\delta} d \mu_{O}(\xi) \\
= & c\left[\int_{B\left(\omega, x_{k}\right)} g(\xi)\left(\frac{x_{k}}{\left|\xi-x^{\prime}\right|^{2}+x_{k}^{2}}\right)^{\delta} d \mu_{O}(\xi)+\right. \\
& \left.+\sum_{j \geq 0} \int_{B\left(\omega, 2^{j+1} x_{k}\right) \backslash B\left(\omega, 2^{j} x_{k}\right)} g(\xi)\left(\frac{x_{k}}{\left|\xi-x^{\prime}\right|^{2}+x_{k}^{2}}\right)^{\delta} d \mu_{O}(\xi)\right] \\
\leq & c\left[\frac{1}{x_{k}^{\delta}} \int_{B\left(\omega, x_{k}\right)} g(\xi) d \mu_{O}(\xi)+\sum_{j \geq 0} \frac{1}{\left(c 2^{2 j} x_{k}^{2}\right)^{\delta}} \int_{B\left(\omega, 2^{j+1} x_{k}\right) \backslash B\left(\omega, 2^{j} x_{k}\right)} g(\xi) d \mu_{O}(\xi)\right] \\
\leq & c\left[\frac{1}{\mu_{O}\left(B\left(\omega, x_{k}\right)\right)} \int_{B\left(\omega, x_{k}\right)} g(\xi) d \mu_{O}(\xi)+\sum_{j \geq 0} \frac{2^{-j}}{\mu_{O}\left(B\left(\omega, x_{k}^{j+1}\right)\right)} \int_{B\left(\omega, 2^{j+1} x_{k}\right)} g(\xi) d \mu_{O}(\xi)\right] \\
\leq & c N g(\omega)\left(1+\sum_{j \geq 0} 2^{-j}\right) \leq c N g(\omega) .
\end{aligned}
$$

The constant $c$ actually depends on $\alpha$ because we used that for $x \in C_{\alpha}(\omega)$ and $\xi \in B\left(\omega, 2^{j+1} x_{k}\right) \backslash B\left(\omega, 2^{j} x_{k}\right)$ we have $\left|\xi-x^{\prime}\right|^{2}+x_{k}^{2} \geq c 2^{2 j} x_{k}^{2}$. It can be shown that $c$ is bounded by $(\tan \alpha)^{2 \delta}$.

We can now finish the proof of Theorem 6.2. Since $\left\|\mu_{\gamma O}\right\|=1$ and $\lim _{x \rightarrow \omega} e^{-\delta B^{K}(x, z)}=0$ for all $z \neq \omega$, the claim is true for continuous functions. Suppose now $f \in L^{1}\left(\partial \mathbb{H}^{k}, \mu_{O}\right)$, and let $f_{j} \rightarrow f$ be a sequence of continuous function converging to $f \mu_{O}$-almost everywhere and in $L^{1}$. We have

$$
\begin{aligned}
\left|\int_{\partial \mathbb{H}^{k}} f(\xi) d \mu_{\gamma O}(\xi)-f(\omega)\right| & \leq\left|\int_{\partial \mathbb{H}^{k}} f(\xi)-\varphi_{j}(\xi) d \mu_{\gamma O}(\xi)\right|+ \\
& +\left|\int_{\partial \mathbb{H}^{k}} f_{j}(\xi) d \mu_{\gamma O}(\xi)-f_{j}(\omega)\right|+\left|f_{j}(\omega)-f(\omega)\right|
\end{aligned}
$$

The second summand of the second term goes to zero as $\gamma O \rightarrow \omega$ because $f_{j}$ is continuous. For $\mu_{O}$-almost all $\omega$ the last summand can be chosen arbitrarily small because $f_{j} \rightarrow f \mu_{O}$-almost everywhere. By Lemma 6.4 the first summand of the second term is bounded by $c N\left(f_{j}-f\right)(\omega)$ on each cone $C_{\alpha}(\omega)$, the constant $c$ depending on $\alpha$. The Hardy Littlewood operator is 
bounded from $L^{1}$ to $L^{1, \infty}$ (see for example [Ste70]), that is, for all $g \in L^{1}$ and $\epsilon>0$

$$
\mu_{O}\left(\left\{\omega \in \partial \mathbb{H}^{k}:|N g(\omega)| \geq \epsilon\right\}\right) \leq \frac{c\|g\|_{L^{1}}}{\epsilon}
$$

Let $A_{j}^{\epsilon}=\left\{\omega \in \partial \mathbb{H}^{k}:\left|N\left(f_{j}-f\right)(\omega)\right| \geq \epsilon\right\}$. Since $f_{j} \rightarrow f$ in $L^{1}$, for all $\varepsilon$ the measure $\mu_{O}\left(A_{j}^{\epsilon}\right)$ goes to zero. This is equivalent to say that the characteristic function $\chi_{A_{j}^{\epsilon}} \rightarrow 0$ in $L^{1}$. Then, up to pass to subsequences, $\chi_{A_{j}^{\epsilon}} \rightarrow 0 \mu_{O}$-almost everywhere, that is, for $\mu_{O}$-almost all $\omega$ the quantity $\left|N\left(f_{j}-f\right)(\omega)\right| \leq \epsilon$ eventually on $j$. We have so proved that, for each $\alpha \in$ $(0, \pi / 2)$ there exist a negligible set $Z_{\alpha}^{\epsilon}$ such that, for all $\omega \in \partial \mathbb{H}^{k} \backslash Z_{\alpha}^{\epsilon}$, the quantity $\left|\int_{\partial \mathbb{H}^{k}} f(\xi) d \mu_{\gamma O}(\xi)-f(\omega)\right|$ is small than or equal to $\epsilon$ as $\gamma O$ converges to $\omega$ through $C_{\alpha}(\omega)$. The thesis now follows setting

$$
Z=\bigcup_{\substack{\alpha \in \mathbb{Q} \cap(0, \pi / 2) \\ 0<\epsilon \in \mathbb{Q}}} Z_{\alpha}^{\epsilon} .
$$

\section{Measurable, equivariant Peano curves}

In this sections we prove Theorem [1.8, Corollary 1.9, Theorem [1.10] and Corollary 1.11. We keep here the notations of previous sections. In particular, if not specified, $\Gamma$ is a discrete group of $\operatorname{Isom}\left(\mathbb{H}^{\mathrm{k}}\right), \rho: \Gamma \rightarrow \operatorname{Isom}\left(\mathbb{H}^{\mathrm{n}}\right)$ is a representation whose image is not elementary, $\left\{\mu_{x}\right\}$ is the family of PattersonSullivan measure, and $\left\{\lambda_{z}\right\}_{\mathbf{z} \in \partial \mathbb{H}^{k}}$ is a family of developing measures for $\rho$.

The following two lemmas collect some ergodic properties of $\Gamma$ that we need in the sequel (see [Yue96b, Theorem A] and [Nic89, Theorem 6.3.6] for the proofs).

Lemma 7.1 Any non-elementary discrete group $\Gamma$ acts ergodically on $\Lambda_{c}$ w.r.t. the measure $\mu_{O}$. Moreover, $\Lambda_{c}$ has either zero or full $\mu_{O}$-measure. Finally the following are equivalent:

1. $\Lambda_{c}$ has full measure.

2. $\Gamma$ diverges at $\delta(\Gamma)$.

3. The geodesic flow is ergodic. 
Lemma 7.2 The group $\Gamma$ acts ergodically on $\partial \mathbb{H}^{k} \times \partial \mathbb{H}^{k}$ w.r.t. $\mu_{O} \times \mu_{O}$ if and only if $\delta(\Gamma)=k-1$ and $\Gamma$ diverges at $k-1$.

To begin with, we prove a couple of lemmas we need for the proof of Theorem 1.8

Lemma 7.3 The subset of $\partial \mathbb{H}^{k}$ consisting of the points $z$ such that $\lambda_{z}$ is the sum of two Dirac deltas with equal weights is $\mu_{O}$-measurable.

Proof. Let $B(\theta, r)$ denotes the ball of center $\theta \in \partial \mathbb{H}^{n}$ and radius $r$ in some metric of $\partial \mathbb{H}^{n}$, and let $Q$ be a countable, dense subset of $\partial \mathbb{H}^{n}$.

For any open set $B \subset \partial \mathbb{H}^{n}$, the function $z \mapsto \lambda_{z}(B)$ is $\mu_{O}$-measurable. It follows that the function

$$
z \mapsto \inf _{0<r_{1}, r_{2} \in \mathbb{Q}}\left(\sup _{\theta_{1}, \theta_{2} \in Q} \lambda_{z}\left(B\left(\theta_{1}, r_{1}\right) \cup B\left(\theta_{2}, r_{2}\right)\right)\right)
$$

is $\mu_{O}$-measurable. The pre-image of 1 , which therefore is a $\mu_{O}$-measurable set, is the set of points $z$ such that the support of $\lambda_{z}$ contains at most two points. Similarly, the following sets are $\mu_{O}$-measurable

$$
\begin{gathered}
\left\{z \in \partial \mathbb{H}^{k}: \lambda_{z} \text { is concentrated on one point }\right\} \\
\left\{z \in \partial \mathbb{H}^{k}: \lambda_{z} \text { has an atom of weight } \frac{1}{2}\right\}
\end{gathered}
$$

and the claim follows.

Lemma 7.4 For all $\mu_{O}$-measurable set $A \subset \partial \mathbb{H}^{k}$ the set

$$
\mathcal{O}(A)=\left\{(x, y) \in \partial \mathbb{H}^{k} \times \partial \mathbb{H}^{k}: \exists \gamma \in \Gamma: \gamma(x), \gamma(y) \in A\right\}
$$

is $\mu_{O} \times \mu_{O}$-measurable.

Proof. Clearly, it is sufficient to show that the function

$$
(x, y) \mapsto \#\{\gamma \in \Gamma: \gamma(x), \gamma(y) \in A\}
$$

is $\mu_{O} \times \mu_{O}$-measurable. The pre-image of $(n, \infty]$ is the set

$$
\begin{aligned}
& \bigcup \quad\left(\left(\left(\gamma_{1}(A) \times \gamma_{1}(A)\right) \cap \cdots \cap\left(\left(\gamma_{n}(A) \times \gamma_{n}(A)\right)\right)\right.\right. \\
& \gamma_{1}, \ldots, \gamma_{n} \in \Gamma \\
& \gamma_{1} \neq \cdots \neq \gamma_{n}
\end{aligned}
$$

which is a countable union of countable measurable sets, and therefore it is measurable. 
Lemma 7.5 Suppose that $\delta(\Gamma)=k-1$ and that $\Gamma$ diverges at $k-1$. If $\mu_{O}(A)>0$, then $\mathcal{O}(A)$ has full measure.

Proof. Since $\mu_{O}(A)>0$, then $A \times A$ has positive measure. Thus, $\mathcal{O}(A)$ has positive measure because it contains $A \times A$. Moreover, it is readily checked that $\mathcal{O}(A)$ is $\Gamma$-invariant. By Lemma 7.2 the action of $\Gamma$ on $\partial \mathbb{H}^{k} \times \partial \mathbb{H}^{k}$ is ergodic, whence $\mathcal{O}(A)$ has full measure.

Now we prove Theorem 1.8. We recall that, by hypothesis, $\delta(\Gamma)=k-1$ and $\Gamma$ diverges at $k-1$.

Proof of Theorem 1.8, By Lemmas [7.1 and 7.2 the conical limit set has full measure in $\partial \mathbb{H}^{k}$ and $\Gamma$ acts ergodically on $\partial \mathbb{H}^{k}$. Let $E$ be the set of points $z \in \partial \mathbb{H}^{k}$ such that $\lambda_{z}$ is the sum of two deltas with equal weights. We have to prove that $\mu_{O}(E)=0$. Clearly, the set $E$ is $\Gamma$-invariant, and by Lemma $[7.3$ it is measurable. Therefore it has either zero or full $\mu_{O}$-measure.

Suppose that $E$ has full $\mu_{O^{-}}$-measure. Then it is well defined and $\mu_{O^{-}}$ measurable the map

$$
\begin{aligned}
f: \partial \mathbb{H}^{k} & \rightarrow\left(\partial \mathbb{H}^{n} \times \partial \mathbb{H}^{n}\right) / \mathfrak{S}_{2} \\
z & \mapsto \text { support of } \lambda_{z} .
\end{aligned}
$$

By Lusin theorem (see for example AFP00]) for all $\varepsilon>0$ there exists a compact set $A \subset \partial \mathbb{H}^{k}$, with $\mu_{O}\left(\partial \mathbb{H}^{k} \backslash A\right)<\varepsilon$ and such that the restriction of $f$ to $A$ is continuous. By Lemma [7.5, for any density-point $x$ of $A$, for $\mu_{O} \times \mu_{O}$-almost all $\left(z_{1}, z_{2}\right) \in \partial \mathbb{H}^{k} \times \partial \mathbb{H}^{k}$ there exists a sequence $\left\{\gamma_{i}\right\} \subset \Gamma$ such that for $j=1,2$

$$
\gamma_{i}\left(z_{j}\right) \in A \quad \text { and } \quad \gamma_{i}\left(z_{j}\right) \rightarrow x .
$$

Therefore, for $j=1,2$

$$
f\left(\gamma_{i}\left(z_{j}\right)\right) \rightarrow f(x)
$$

Up to pass to subsequences, $\rho\left(\gamma_{i}\right)$ converges either to an isometry of $\mathbb{H}^{n}$ or to a quasi-constant $C_{a}^{b}-$ a quasi-constant is a map such that $C_{a}^{b}(p)=b$ for all points $p \neq a$ of $\overline{\mathbb{H}}^{n}$ - where the convergence is uniform on compact sets not containing $a$ (see for example Kap01).

Let $\{\theta, \omega\}$ be the support of $\lambda_{x}$, that is $\lambda_{x}=\frac{\delta_{\theta}+\delta_{\omega}}{2}$, and for $j=1,2$ let $\left\{\xi_{j}, \zeta_{j}\right\}$ be the support of $\lambda_{z_{j}}$.

If $\rho\left(\gamma_{i}\right)$ converges to an isometry $\psi$, then for $j=1,2$

$$
f\left(\gamma_{i}\left(z_{j}\right)\right)=\rho\left(\gamma_{i}\right)\left\{\xi_{j}, \zeta_{j}\right\} \rightarrow\left\{\psi\left(\xi_{j}\right), \psi\left(\zeta_{j}\right)\right\}
$$


Since $f\left(\gamma_{i}\left(z_{j}\right)\right) \rightarrow f(x)=\{\theta, \omega\}$, we get $f\left(z_{1}\right)=f\left(z_{2}\right)$. On the other side, if $\rho\left(\gamma_{i}\right) \rightarrow C_{a}^{b}$ and if $\xi_{j} \neq a \neq \zeta_{j}$, we get

$$
f\left(\gamma_{i}\left(z_{j}\right)\right) \rightarrow\{b, b\} \neq f(x) .
$$

We have so proved that for $\mu_{O} \times \mu_{O}$-almost all $\left(z_{1}, z_{2}\right) \in \partial \mathbb{H}^{k} \times \partial \mathbb{H}^{k}$ the supports of $\lambda_{\mathbf{z}_{1}}$ and $\lambda_{z_{2}}$ share at least one point. Whence, using Fubini's theorem and Lemma [7.5. it follows that there exists $\zeta \in \partial \mathbb{H}^{n}$ such that for $\mu_{O}$-almost all $z \in \partial \mathbb{H}^{k}$, the support of $\lambda_{z}$ contains $\zeta$. For $z \in \partial \mathbb{H}^{k}$, let $\xi(z)$ denote the other point of the support of $\lambda_{z}$, that is

$$
f(z)=\{\zeta, \xi(z)\}
$$

For each $\gamma \in \Gamma$, for $\mu_{O}$-almost all $z$ we have $f(z)=\{\zeta, \xi(z)\}$ and

$$
\{\rho(\gamma) \zeta, \rho(\gamma) \xi(z)\}=\rho(\gamma)(f(z))=f(\gamma z)=\{\zeta, \xi(\gamma z)\}
$$

Whence we have

Either: the set $\{\zeta\}$ is $\rho(\Gamma)$-invariant.

Or: there exists $\gamma \in \Gamma$ such that $\rho(\gamma) \zeta \neq \zeta$, which implies that $\xi(z)=$ $\rho(\gamma)^{-1}(\zeta)$ does not depend on $z$. Therefore the set $f(z)$ does not depend on $z$ and it is $\rho(\Gamma)$-invariant.

In both cases the image of $\rho$ is elementary, which contradicts the hypotheses. It follows that the set $E$ cannot have full $\mu_{O}$-measure.

We conclude this section by proving Corollary [1.9] Theorem [1.10] and Corollary 1.11

Proof of Corollary 1.9. It is well-known (see for example Kap01) that if $\mathbb{H}^{k} / \Gamma$ is a complete hyperbolic manifold of finite volume, then $\delta(\Gamma)=k-1$ and $\Lambda_{c}$ has full-measure. Then, by Lemmas [7.1 and [7.2, the hypotheses of Theorem 1.8 are satisfied, and the first claim follows. If in addiction $\rho$ is discrete and faithful, the $\rho$-equivariance implies the second claim.

Proof of Theorem 1.10. The first claim is an immediate corollary of Theorems [1.7 and 1.8. Now, let $\varphi=\bar{F} \circ \bar{G}: \partial \mathbb{H}^{k} \rightarrow \partial \mathbb{H}^{k}$. The map $\varphi$ is clearly $\Gamma$-equivariant and $\mu_{O}$-measurable. It follows that the set $\{x: \varphi(x)=x\}$ is $\mu_{O}$-measurable and $\Gamma$-invariant. Thus, by ergodicity (Lemma 17.1), it has 
either zero or full measure, and we are claiming that it has full measure. Arguing as in the proof of Theorem 1.8, one can show that there exists a positive-measure set $A$ such that for all $x \in A$ and for $\mu_{O} \times \mu_{O}$-almost all $\left(z_{1}, z_{2}\right) \in \partial \mathbb{H}^{k} \times \partial \mathbb{H}^{k}$ there exists a sequence $\left\{\gamma_{i}\right\} \subset \Gamma$ such that for $j=1,2$

$$
\gamma_{i}\left(z_{j}\right) \rightarrow x \quad \text { and } \quad \varphi\left(\gamma_{i}\left(z_{j}\right)\right) \rightarrow \varphi(x) .
$$

Since $\Gamma$ is discrete, up to pass to subsequences, $\gamma_{i}$ converges to a quasiconstant $C_{a}^{b}$. If $z_{1} \neq a \neq \varphi\left(z_{1}\right)$, then

$$
\gamma_{i}\left(z_{1}\right) \rightarrow b \quad \text { and } \quad \gamma_{i}\left(\varphi\left(z_{1}\right)\right) \rightarrow b
$$

since $\gamma_{i}\left(\varphi\left(z_{1}\right)\right)=\varphi\left(\gamma_{i}\left(z_{1}\right)\right) \rightarrow \varphi(x)$, we get $b=x=\varphi(x)$. The same holds if we replace $z_{1}$ with $z_{2}$.

Suppose now that for $j=1,2$ either $z_{j}=a$ or $\varphi\left(z_{j}\right)=a$. Since $\Gamma$ is non-elementary, $\mu_{O}$ is not purely atomic. In particular it is not restrictive to suppose $z_{1} \neq z_{2}$ and

$$
z_{1}=a \quad \varphi\left(z_{1}\right) \neq a \quad z_{2} \neq a \quad \varphi\left(z_{2}\right)=a .
$$

Therefore, $\varphi\left(\gamma_{i}\left(z_{1}\right)\right)=\gamma_{i}\left(\varphi\left(z_{1}\right)\right) \rightarrow b$, and then $b=\varphi(x)$. On the other side, $\gamma_{i}\left(z_{2}\right) \rightarrow b$, whence $x=b$, which implies $x=\varphi(x)$. We have so proved that $A \subset\{x: \varphi(x)=x\}$ which then has positive measure and whence full measure. It follows that $\bar{F} \circ \bar{G}=\operatorname{Id}_{\mathbb{H}^{k}} \mu_{O^{-}}$almost everywhere. Clearly the same holds replacing $\bar{F} \circ \bar{G}$ with $\bar{G} \circ \bar{F}$.

Proof of Corollary 1.11. Clearly, $\Gamma$ satisfies the hypotheses of Theorem 1.10, Moreover, by Lemma 7.1, Lemma 7.2 and [Can93, Corollary 2], also $\pi_{1}(F)<$ $\pi_{1}(M)<\operatorname{Isom}\left(H^{3}\right)$ satisfies the hypotheses of Theorem 1.10.

\section{References}

[AFP00] Luigi Ambrosio, Nicola Fusco, and Diego Pallara. Functions of bounded variation and free discontinuity problems. Oxford Mathematical Monographs. The Clarendon Press Oxford University Press, New York, 2000.

[BCG95] Gérard Besson, Gilles Courtois, and Sylvestre Gallot. Entropies et rigidités des espaces localement symétriques de courbure strictement négative. Geom. Funct. Anal., 5(5):731-799, 1995. 
[BCG96] Gérard Besson, Gilles Courtois, and Sylvestre Gallot. Minimal entropy and Mostow's rigidity theorems. Ergodic Theory Dynam. Systems, 16(4):623-649, 1996.

[BCG99] Gérard Besson, Gilles Courtois, and Sylvestre Gallot. Lemme de Schwarz réel et applications géométriques. Acta Math., 183(2):145169, 1999.

[BP92] Riccardo Benedetti and Carlo Petronio. Lectures on hyperbolic geometry. Universitext. Springer-Verlag, Berlin, 1992.

[Can93] Richard D. Canary. Ends of hyperbolic 3-manifolds. J. Amer. Math. Soc., 6(1):1-35, 1993.

[CT89] James W. Cannon and William P. Thurston. Group invariant Peano curves. Preprint, 1989.

[DE86] Adrien Douady and Clifford J. Earle. Conformally natural extension of homeomorphisms of the circle. Acta Math., 157(1-2):23-48, 1986.

[DM78] Claude Dellacherie and Paul-André Meyer. Probabilities and potential, volume 29 of North-Holland Mathematics Studies. NorthHolland Publishing Co., Amsterdam, 1978.

[Dun99] Nathan M. Dunfield. Cyclic surgery, degrees of maps of character curves, and volume rigidity for hyperbolic manifolds. Invent. Math., 136(3):623-657, 1999.

[FK04] Stefano Francaviglia and Benjamin Klaff. Maximal volume representations are Fuchsian. In preparation, 2004.

[Fra04] Stefano Francaviglia. Hyperbolic volume of representations of fundamental groups of cusped 3-manifolds. Int. Math. Res. Not., (9):425-459, 2004.

[Kap01] Michael Kapovich. Hyperbolic manifolds and discrete groups, volume 183 of Progress in Mathematics. Birkhäuser Boston Inc., Boston, MA, 2001.

[Kla] B. Klaff. Boundary slopes of knots in closed 3-manifolds with cyclic fundamental group. PhD thesis, University Illinois-Chicago, 2003.

[Min94] Yair N. Minsky. On rigidity, limit sets, and end invariants of hyperbolic 3-manifolds. J. Amer. Math. Soc., 7(3):539-588, 1994. 
[Nic89] Peter J. Nicholls. The ergodic theory of discrete groups, volume 143 of London Mathematical Society Lecture Note Series. Cambridge University Press, Cambridge, 1989.

[Som95] Teruhiko Soma. Equivariant, almost homeomorphic maps between $S^{1}$ and $S^{2}$. Proc. Amer. Math. Soc., 123(9):2915-2920, 1995.

[Sou00] J. Souto. Minimal volume and Minimal entropy. PhD thesis, Bonn, 2000 .

[Ste70] Elias M. Stein. Singular integrals and differentiability properties of functions. Princeton Mathematical Series, No. 30. Princeton University Press, Princeton, N.J., 1970.

[Sul84] Dennis Sullivan. Entropy, Hausdorff measures old and new, and limit sets of geometrically finite Kleinian groups. Acta Math., 153(3-4):259-277, 1984.

[Thu79] W. P. Thurston. The geometry and topology of 3-manifolds. Mimeographed notes. Princeton University Mathematics Department, 1979.

[Yue96a] Chengbo Yue. Dimension and rigidity of quasi-Fuchsian representations. Ann. of Math. (2), 143(2):331-355, 1996.

[Yue96b] Chengbo Yue. The ergodic theory of discrete isometry groups on manifolds of variable negative curvature. Trans. Amer. Math. Soc., 348(12):4965-5005, 1996.

Stefano Francaviglia

Dipartimento di Matematica Applicata "Ulisse Dini"

Via Bonanno 25-b, I-56126 Pisa, Italy.

e-mail: francavi@sns.it 